\title{
The Use of Epoxy Silanes on Montmorillonite: An Effective Way to Improve Thermal and Rheological Properties of PLA/MMT Nanocomposites Obtained via "In Situ" Polymerization
}

\author{
Valentina Sabatini, ${ }^{1,2}$ Hermes Farina, ${ }^{1,2}$ Luca Basilissi, ${ }^{1}$ \\ Giuseppe Di Silvestro, ${ }^{1,2}$ and Marco A. Ortenzi ${ }^{1,2}$ \\ ${ }^{1}$ Department of Chemistry, University of Milan, Via Golgi 19, 20133 Milan, Italy \\ ${ }^{2}$ CRC Materiali Polimerici (LaMPo), Department of Chemistry, University of Milan, Via Golgi 19, 20133 Milan, Italy
}

Correspondence should be addressed to Marco A. Ortenzi; marco.ortenzi@unimi.it

Received 13 April 2015; Revised 15 June 2015; Accepted 18 June 2015

Academic Editor: Yibing Cai

Copyright (C) 2015 Valentina Sabatini et al. This is an open access article distributed under the Creative Commons Attribution License, which permits unrestricted use, distribution, and reproduction in any medium, provided the original work is properly cited.

Polylactic acid (PLA) nanocomposites were prepared via "in situ" ring opening polymerization (ROP) of lactide using a montmorillonite, Cloisite 15A, employed after surface treatment with 3-Glycidoxypropyltrimethoxysilane. The dispersion of the nanoparticles was checked using Wide Angle X-Ray Scattering (WAXS) and Transmission Electron Microscopy (TEM); both the effects of different amounts of montmorillonite and silane were measured on molecular weights and on thermal and rheological properties, using Size Exclusion Chromatography (SEC), Differential Scanning Calorimetry (DSC), thermogravimetric analyses (TGA), and rheological analyses. It was found that even very low amounts $(0.1 \% \mathrm{w} / \mathrm{w})$ of nanoparticles greatly affect nanocomposites properties. Unmodified montmorillonite tends to decrease molecular weights, deactivating the catalytic system used for ROP of lactide, but when epoxy silane is present molecular weights increase. Melt crystallization temperatures increase with modified nanoparticles, which enhance crystallization process. TGA analyses show that when pure montmorillonite is present, nanocomposites have lower thermal stability with respect to standard PLA; when silane is used thermal stability can get much higher than standard PLA as silane content increases. The rheological behaviour of nanocomposites shows that melt viscosity is far higher than that of standard PLA at low shear rates and also a marked shear thinning behaviour can be achieved.

\section{Introduction}

Polylactic acid (PLA) is an aliphatic polyester derived from renewable resources [1], such as sweet corn or sugar beets. It can be synthesized either by direct condensation of lactic acid or by ring opening polymerization (ROP) of lactic acid cyclic dimer, lactide.

PLA is receiving more and more attention both in scientific and in industrial research fields because it combines a low environmental impact, regarding both raw materials production and end-life disposal, with interesting features; some of them include reduction of $\mathrm{CO}_{2}$ emission in comparison to conventional petroleum-based commodity plastics [2], improvement of farm economies [3], good physical properties and processability [4], and biodegradation to lactic acid, which is not harmful for human body [5]. Moreover, when burned, it produces no nitrogen oxide gases and only onethird of the heat generated by polyolefins [6].

In the last years, PLA has therefore dramatically increased its market share, especially for the production of packaging and disposables. Nevertheless the polymer still suffers from some drawbacks, preventing its use for even more applications; it has poor thermal and mechanical resistance in processing conditions [7] and poor gas barrier properties [8], which limit its applications as food packaging material, a field in which its use, despite a price higher than traditional 
TABLE 1: Key physical properties of Cloisite 15A.

\begin{tabular}{|c|c|c|c|c|}
\hline Modifier structure & Organomodifier & Density $(\mathrm{g} / \mathrm{mL})$ & $d_{001}$-spacing $(\mathrm{nm})$ & $\begin{array}{l}\text { Weight loss on } \\
\text { ignition (wt } \%)\end{array}$ \\
\hline \multicolumn{5}{|l|}{$\mathrm{CH}_{3}$} \\
\hline $\mathrm{H}_{3} \mathrm{C}-\mathrm{N}^{+}-\mathrm{HT}$ & $\begin{array}{l}\text { Dimethyl dehydrogenated } \\
\text { tallow ammonium }\end{array}$ & 1.66 & 3.15 & 43 \\
\hline HT & & & & \\
\hline
\end{tabular}

polyolefins such as PE, PET, and PP, is justified when biodegradability is required [9].

PLA nanocomposites have been investigated as possible solution to the problems evidenced, since they exhibit remarkably improved mechanical, thermal, and flexural properties, heat distortion temperature, and gas permeability compared to those of neat PLA polymers [10-13].

When using minerals with nanoscale dimensions, two important aspects must be considered: the compatibility between polymer and minerals and their dispersion in the matrix. Many nanoparticles have a very high surface/volume ratio that favors the dispersion in the polymeric matrix; thus significant improvements of properties can be obtained even with low loadings of mineral, even up to $0.1 \% \mathrm{w} / \mathrm{w}$, maintaining processing conditions similar to those of standard materials [14].

Within this framework, in the last decades the addition of layered silicate nanoparticles, such as montmorillonite (MMT), to polymers has attracted great attention for the potentiality of these minerals to influence a number of properties: for example, polyamides layered silicate sheets exhibit, even at low filler content ( $1 \% \mathrm{w} / \mathrm{w})$, marked improvements regarding mechanical, thermal, and barrier properties in comparison to the unfilled matrix and to more conventional composites [15].

It is well known that there are two ideal types of nanostructures in polymer/MMT nanocomposites, deriving from intercalation and exfoliation $[16,17]$ of the mineral lamellae. The intercalated structure is formed when a few polymer molecules are inserted between MMT layers and the nanoparticles are therefore "packed." On the other side, complete exfoliation is achieved when the macromolecules separate the lamellae and therefore mineral layers are individually dispersed in polymer matrix. Real morphologies of PLA/MMT nanocomposites rarely contain the two idealized nanostructures; these two structures often coexist in the nanocomposites, but it is believed that the remarkable mechanical and barrier properties of these materials result when exfoliation is dominant [18].

Most of the scientific literature deals with PLA/MMT nanocomposites obtained via extrusion, using high torque twin-screw extruders to achieve an efficient dispersion of the mineral in the polymer matrix $[19,20]$ or via dissolution and reprecipitation [21]; there are only a few works about the "in situ" preparation of PLA/MMT nanocomposites [22, 23] that could result in being a very efficient way to have nanocomposites with high exfoliation degree thanks to the high residential time of the nanoparticles in the polymeric matrix and to the fact that, at the beginning of the polymerization, short macromolecular chains have high mobility and reduced dimensions, allowing them to better exfoliate the mineral lamellae.

The present paper investigates the behaviour of PLA nanocomposites obtained using "in situ" polymerization; nanocomposites were prepared by bulk ring opening polymerization (ROP) of lactide using a MMT, trademark Cloisite $15 \mathrm{~A}$ by Rockwood Additives Inc., modified with a quaternary ammonium salt between the lamellae, that increases the space between each layer promoting exfoliation [24]. Since another issue limiting exfoliation is the high difference of surface energy between the inorganic platelets of MMT and the organic polymeric matrix [25], in the present paper a silane, 3-Glycidoxypropyltrimethoxysilane, was used as coupling agent to modify the surface of MMT in order to have a higher compatibility with the organic matrix and therefore to enhance exfoliation during the polymerization. Surface modification with silanes is well known for traditional mineral fillers such as wollastonite, kaolin, or glass fiber; it has also been used with mineral nanoparticles [26] but it has been not intensively applied on nanoparticles used to create PLA nanocomposites. Just a few examples are present in literature, for example, regarding nanosilica [27], and show that silanes have a marked effect on many properties; the effects of surface modification of MMT were investigated on the molecular weight, thermal properties, and rheological behaviour of PLA/MMT nanocomposites prepared by ROP of lactide using different quantities of silane-modified MMT to understand if the presence of silane, together with the "in situ" synthesis, can lead to improvements in the properties of PLA/MMT nanocomposites in comparison to what is already present in literature.

\section{Experimental}

2.1. Materials and Methods. PLA was synthesized from LLlactide PURASORB L (purity > 99.5\%) purchased from Purac Biomaterials. Tin octanoate [Sn(oct $\left.)_{2}\right]$, pyromellitic dianhydride (PMDA), methanol, chloroform, methylene chloride, and $\mathrm{m}$-cresol were purchased from Sigma Aldrich Co. LLC. Cloisite $15 \mathrm{~A}$ is a montmorillonite modified with an organic ammonium salt; it has an average packed diameter of $13 \mu \mathrm{m}$ and ammonium salt concentration of $125 \mathrm{meq} / 100 \mathrm{~g}$ and was purchased from Rockwood Additives Inc. Key physical properties of this clay, as declared by the producer, are summarized in Table 1. In the text, it will be referred to as MMT. 3-Glycidoxypropyltrimethoxysilane (GF80) was purchased from Wacker Chemie AG. All the reagents were used without further purification. 
2.1.1. Wide Angle X-Ray Scattering (WAXS). Wide Angle XRay Scattering (WAXS) experiments were performed using a Rigaku DMAX-II diffractometer. Diffraction patterns were obtained in the range $5^{\circ}<2 \theta<60^{\circ}$ with $\mathrm{Cu}-\mathrm{K} \alpha$ radiation $(\lambda=1.5405 \AA)$ under the following conditions: $40 \mathrm{kV}, 40 \mathrm{~mA}$, step width $0.02^{\circ}$, time per step $2 \mathrm{sec}$, divergence slit $0.25^{\circ}$, Soller slit $0.04 \mathrm{rad}$, and antiscatter slit $0.5^{\circ}$. X-ray patterns are normalized on the main peak. The basal spacing of the clay was calculated using Bragg's law (equation (1)), where $n$ represents the integer of diffraction order, $\lambda$ is the wavelength of the incident light $(1.5405 \AA), d$ is the lattice spacing, and $\theta$ is the angle of diffraction:

$$
n \lambda=2 d \sin \theta .
$$

2.1.2. Transmission Electron Microscopy (TEM). The dispersion of MMT in the polymeric matrix was evaluated via Transmission Electron Microscopy (TEM): samples were reduced into ultrathin sections of $80 \mathrm{~nm}$ by an Ultracut $\mathrm{E}$ microtome (Reichert), collected onto a 300-mesh copper grid and examined by an EFTEM Leo912ab TEM (Zeiss) operating at $80 \mathrm{kV}$. Digital images were acquired by Esivision CCD$\mathrm{BM} / 1 \mathrm{~K}$ system.

2.1.3. Size Exclusion Chromatography (SEC). The effect of MMT on the molecular weight of the nanocomposites was evaluated using a SEC system having Waters 1515 Isocratic HPLC pump and a four Waters Styragel columns' set (HR3HR4-HR5-HR2) with a UV detector Waters 2487 Dual $\lambda$ Absorbance Detector set at $230 \mathrm{~nm}$ using a flow rate of $1 \mathrm{~mL} /$ min and $60 \mu \mathrm{L}$ as injection volume; samples were prepared dissolving $40 \mathrm{mg}$ of polymer in $1 \mathrm{~mL}$ of anhydrous $\mathrm{CH}_{2} \mathrm{Cl}_{2}$; before the analysis, the solution was filtered with $0.45 \mu \mathrm{m}$ filters. Given the relatively high loading, a check was performed using lower concentration of polymer $(5 \mathrm{mg} / \mathrm{mL})$, in order to verify that no column overloading could be observed. Anyway, higher loadings were preferred as UV signal of PLA is relatively weak.

2.1.4. Differential Scanning Calorimetry (DSC). DSC analyses were conducted using a Mettler Toledo DSC 1, on samples weighting from 5 to $10 \mathrm{mg}$ each. Melting and crystallization temperatures were measured using the following temperature program:

(i) heating from $25^{\circ} \mathrm{C}$ to $200^{\circ} \mathrm{C}$ at $10^{\circ} \mathrm{C} / \mathrm{min}$;

(ii) $5 \mathrm{~min}$ isotherm at $200^{\circ} \mathrm{C}$;

(iii) cooling from $200^{\circ} \mathrm{C}$ to $25^{\circ} \mathrm{C}$ at $10^{\circ} \mathrm{C} / \mathrm{min}$;

(iv) $2 \mathrm{~min}$ isotherm at $25^{\circ} \mathrm{C}$;

(v) heating from $25^{\circ} \mathrm{C}$ to $200^{\circ} \mathrm{C}$ at $10^{\circ} \mathrm{C} / \mathrm{min}$.

The first two cycles (heating and isotherm at $200^{\circ} \mathrm{C}$ ) were conducted to eliminate residual internal stresses deriving from the synthesis. Glass transition temperature $\left(T_{\mathrm{g}}\right)$, specific heat $\left(\Delta C_{p}\right)$, crystallization temperature $\left(T_{c}\right)$, cold crystallization temperature $\left(T_{\mathrm{cc}}\right)$, melting temperature $\left(T_{\mathrm{m}}\right)$, crystallization enthalpy $\left(\Delta H_{c}\right)$, cold crystallization enthalpy $\left(\Delta H_{\mathrm{cc}}\right)$, and melting enthalpy $\left(\Delta H_{\mathrm{m}}\right)$ were determined; a schematic DSC

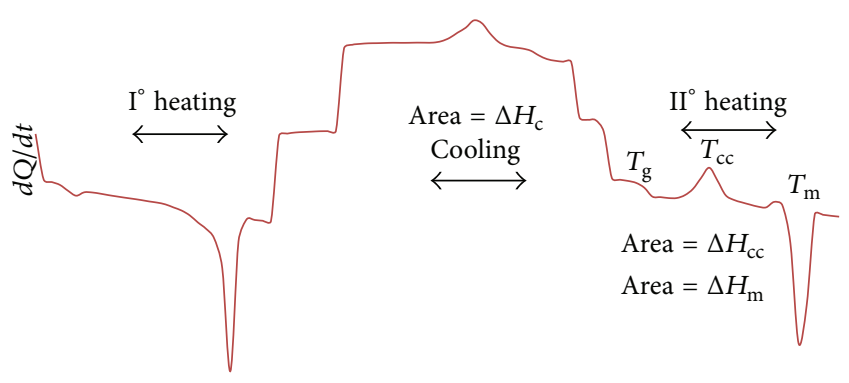

$T\left({ }^{\circ} \mathrm{C}\right)$

FIGURE 1: Schematic representation of calorimetric transitions in a DSC thermogram during heating and cooling cycles.

thermogram, reporting the transitions indicated, is shown in Figure 1.

Room temperature crystallinity $\left(\chi_{\mathrm{c}}\right)$ attributable to cold crystallization of neat PLA and PLA nanocomposites was evaluated taking into account the amount of clay present in nanocomposites using the following equation [28]:

$$
\chi_{\mathrm{c}}=\left(\frac{\Delta H_{\mathrm{c}}}{\Delta H^{\circ} \mathrm{m} *(1-\% \mathrm{wt} \mathrm{MMT} / 100)}\right) * 100,
$$

where $\Delta H_{c}$ is the specific crystallization enthalpy of the sample, $\Delta H^{\circ} \mathrm{m}$ is the melting enthalpy of the $100 \%$ crystalline polymeric matrix (93 J/g for PLA [29]), and \%wt MMT is the weight percentage of MMT.

2.1.5. Thermogravimetric Analyses (TGA). TGA were performed using a TGA 4000 Perkin Elmer instrument; tests on the polymers have been conducted under nitrogen atmosphere on samples weighting from 5 to $10 \mathrm{mg}$ with a program that provides single heating cycle from $30^{\circ} \mathrm{C}$ to $600^{\circ} \mathrm{C}$ at $10^{\circ} \mathrm{C} / \mathrm{min}$. The analyses of nanoparticles were conducted under nitrogen atmosphere heating from $30^{\circ} \mathrm{C}$ to $900^{\circ} \mathrm{C}$ at $10^{\circ} \mathrm{C} / \mathrm{min}$.

2.1.6. Rheological Curves. Rheological analyses, conducted using frequency sweep experiments, were performed with a Physica MCR 300 rotational rheometer with a parallel plate geometry $(\phi=25 \mathrm{~mm}$, distance between plates $=1 \mathrm{~mm})$. Linear viscoelastic regimes of neat PLA and PLA nanocomposites were studied; strain was set equal to $5 \%$ and curves of complex viscosity as function of frequency were recorded, taking 30 points ranging from $100 \mathrm{~Hz}$ to $0.1 \mathrm{~Hz}$ with a logarithmic progression, at $190^{\circ} \mathrm{C}$; in this paper shear rate but not angular frequency is reported on the $x$-axis.

2.2. Surface Modification of MMT. In order to improve the compatibility between the polymeric matrix and the inorganic surface of MMT, organosilane having epoxy functional groups, 3-Glycidoxypropyltrimethoxysilane (GF80), was used as coupling agent to modify the surface of the mineral.

Reaction between silane and MMT is obtained via placing nanoparticles in a one-neck flask in methanol under magnetic stirring, using a MMT/methanol ratio of $1 / 10 \mathrm{w} / \mathrm{w}$ 
TABLE 2: Amount of silane present on Cloisite 15A surface determined via titration.

\begin{tabular}{lc}
\hline Silane added (w/w \% on MMT) & $\mathrm{meq} / \mathrm{kg}$ \\
\hline $2.0 \%$ GF80 & 119 \\
$7.5 \%$ GF80 & 380 \\
$15.0 \%$ GF80 & 712 \\
\hline
\end{tabular}

at room temperature; different amounts of silane are then introduced depending on the desired degree of modification. In this work three different quantities of silane, $2 \%, 7.5 \%$, and $15 \% \mathrm{w} / \mathrm{w}$ on the nanoparticles, have been used. The addition is performed slowly, in about 30 minutes regardless of the quantity of silane, to avoid the formation of aggregates and to favour its good dispersion; the solution is kept under vigorous stirring for 12 hours at room temperature. The solvent is removed by evaporation under nitrogen flux and then the system is heated at $120^{\circ} \mathrm{C}$ under vacuum (about 4 mbar) for 2 hours. Nanoparticles are then chopped using a mortar to obtain a fine powder. An isothermal TGA at $70^{\circ} \mathrm{C}$ under nitrogen flow running for 180 minutes was performed to check the absence of residual solvent on the nanoparticles.

2.2.1. Quantitative Determination of the Silane. The amount of GF80 effectively present on the MMT surface was determined by potentiometric titration of the tertiary amine deriving from the reaction between the epoxy groups and $\mathrm{N}, \mathrm{N}^{\prime}$-dibutylamine. The GF80-modified MMT was dispersed in a solution of $\mathrm{N}, \mathrm{N}^{\prime}$-dibutylamine in methylene chloride (1 gram of MMT was dispersed in a $50 \mathrm{~mL}$ solution of methylene chloride containing 1 gram of $\mathrm{N}, \mathrm{N}^{\prime}$-dibutylamine); the dispersion was stirred at room temperature for $12 \mathrm{~h}$ and then the solvent and the free amine were evaporated under vacuum at $40^{\circ} \mathrm{C}$ for 1 hour. MMT was then dispersed in hot $\mathrm{m}$-cresol and titrated with $\mathrm{HCl} 0.01 \mathrm{~N}$ in methanol. In Table 2 the amount of silane measured via potentiometric titration of the terminal groups is shown.

2.3. Synthesis of PLA and PLA/MMT Nanocomposites. PLA and PLA/MMT nanocomposites were synthesized from LLlactide in bulk according to the following procedure; LLlactide $(50 \mathrm{~g})$ and tin octanoate $(0.3 \% \mathrm{w} / \mathrm{w}$ on lactide $-0.15 \mathrm{~g}$; for PLA13: $3 \% \mathrm{w} / \mathrm{w}$ on lactide $-1.5 \mathrm{~g}$ ) added as catalyst were introduced in a $250 \mathrm{~mL}$ three-neck flask. Slow nitrogen flow was used to ensure the presence of inert atmosphere during the polymerization; mechanical stirring was provided $(100 \mathrm{rpm})$. For the synthesis of nanocomposite PLAs, different quantities of MMT, namely, $1.0 \% \mathrm{w} / \mathrm{w}$ (0.5 grams) $-0.5 \%$ $\mathrm{w} / \mathrm{w}$ (0.25 grams) $-0.1 \% \mathrm{w} / \mathrm{w}$ (0.05 grams), for PLA13 5\% $\mathrm{w} / \mathrm{w}$ (2.5 grams), on lactide, were introduced in the feed together with the other reagents. Reactions were conducted in a closed oven at $180^{\circ} \mathrm{C}$ for 2 hours, using mechanical stirring $(150 \mathrm{rpm})$. At the end of the reaction, the polymer was left in the flask under nitrogen flow and slowly cooled at room temperature; a solid polymer was obtained. PLA nanocomposites containing PMDA were synthesized using the same experimental method and conditions described before adding $0.1 \% \mathrm{w} / \mathrm{w}$ MMT (0.05 g) and PMDA in the feed
TABLE 3: List of samples synthesized.

\begin{tabular}{lcccc}
\hline Sample & $\begin{array}{c}\text { GF80 } \\
(\% \text { w/w on } \\
\text { MMT })\end{array}$ & $\begin{array}{c}\text { MMT } \\
\text { \% w/w on } \\
\text { lactide })\end{array}$ & $\begin{array}{c}\text { Tin octanoate } \\
\text { (\% w/w on } \\
\text { lactide })\end{array}$ & $\begin{array}{c}\text { PMDA } \\
(\mathrm{g})\end{array}$ \\
\hline STD & - & - & 0.3 & - \\
PLA1 & - & 1 & 0.3 & - \\
PLA2 & - & 0.5 & 0.3 & - \\
PLA3 & - & 0.1 & 0.3 & - \\
PLA4 & 2 & 1 & 0.3 & - \\
PLA5 & 2 & 0.5 & 0.3 & - \\
PLA6 & 2 & 0.1 & 0.3 & - \\
PLA7 & 7.5 & 1 & 0.3 & - \\
PLA8 & 7.5 & 0.5 & 0.3 & - \\
PLA9 & 7.5 & 0.1 & 0.3 & - \\
PLA10 & 15 & 1 & 0.3 & - \\
PLA11 & 15 & 0.5 & 0.3 & - \\
PLA12 & 15 & 0.1 & 0.3 & - \\
PLA13 & - & 5 & 3 & $7.77 * 10^{-3}$ \\
PLA14 & - & 0.1 & 0.3 & $7.77 * 10^{-3}$ \\
PLA15 & 15 & 0.1 & 0.3 & \\
\hline
\end{tabular}

$\left(3.56 * 10^{-5} \mathrm{~mol}-7.77 * 10^{-3} \mathrm{~g}\right)$, together with the other reagents. After the syntheses, all samples underwent Solid State Polymerization (SSP) at $150^{\circ} \mathrm{C}$ for 12 hours under vacuum (about $4 \mathrm{mbar}$ ); all the analyses were conducted on the samples obtained after SSP.

2.4. Film Casting. Films for WAXS analyses were obtained from a chloroform solution; 10 grams of polymer was dissolved in 50 grams of $\mathrm{CHCl}_{3}$. The solution was cast on a glass surface and the solvent was evaporated at room temperature and pressure overnight. The absence of residual solvent in the films was checked via isothermal TGA ( 2 hours at $80^{\circ} \mathrm{C}$ under nitrogen flow). Film thickness was evaluated by Nikon eclipse ME600 optical microscope with Nikon digital camera light DS-Fil, software Nis-Elements BR, and magnification 50x, and it was in the range $60-90 \mu \mathrm{m}$.

\section{Results and Discussion}

A list of the samples synthesized, where STD is a standard PLA, is provided in Table 3.

3.1. WAXS Analyses. Crystalline peaks of MMT powder with different amounts of GF80 $(2 \%-7.5 \%-15 \%)$ were studied in order to understand if silane contributes to increasing the interlayer space between the lamellae. Neat MMT exhibits four main diffraction peaks: the major one at $2 \theta=19.77^{\circ}$ and three minor ones at $7.19^{\circ}, 35.31^{\circ}$, and $54.24^{\circ}$, attributed to the reflections of the four crystal planes of MMT. When silane is present, all the four peaks are still visible (Table 4) and each of them has the tendency to shift to lower angles in comparison to pure MMT, as Figure 2 evidences (only peak 2 is almost unaffected), promoting exfoliation. 
TABLE 4: $2 \theta$ values of neat MMT and modified MMT.

\begin{tabular}{lcccc}
\hline $\begin{array}{l}\text { Diffraction } \\
\text { peak }\end{array}$ & $\begin{array}{c}\text { Neat MMT } \\
2 \theta\left[{ }^{\circ}\right]\end{array}$ & $\begin{array}{c}\text { MMT - 2\% } \\
\text { w/w GF80 } \\
2 \theta\left[{ }^{\circ}\right]\end{array}$ & $\begin{array}{c}\text { MMT - 7.5\% } \\
\text { w/w GF80 } \\
2 \theta\left[{ }^{\circ}\right]\end{array}$ & $\begin{array}{c}\text { MMT - 15\% } \\
\text { w/w GF80 } \\
2 \theta\left[{ }^{\circ}\right]\end{array}$ \\
\hline 1 & 7.19 & 6.87 & 6.87 & 6.47 \\
2 & 19.77 & 19.73 & 19.79 & 19.76 \\
3 & 35.31 & 35.06 & 35.12 & 34.86 \\
4 & 54.24 & 53.85 & 54.12 & 53.98 \\
\hline
\end{tabular}

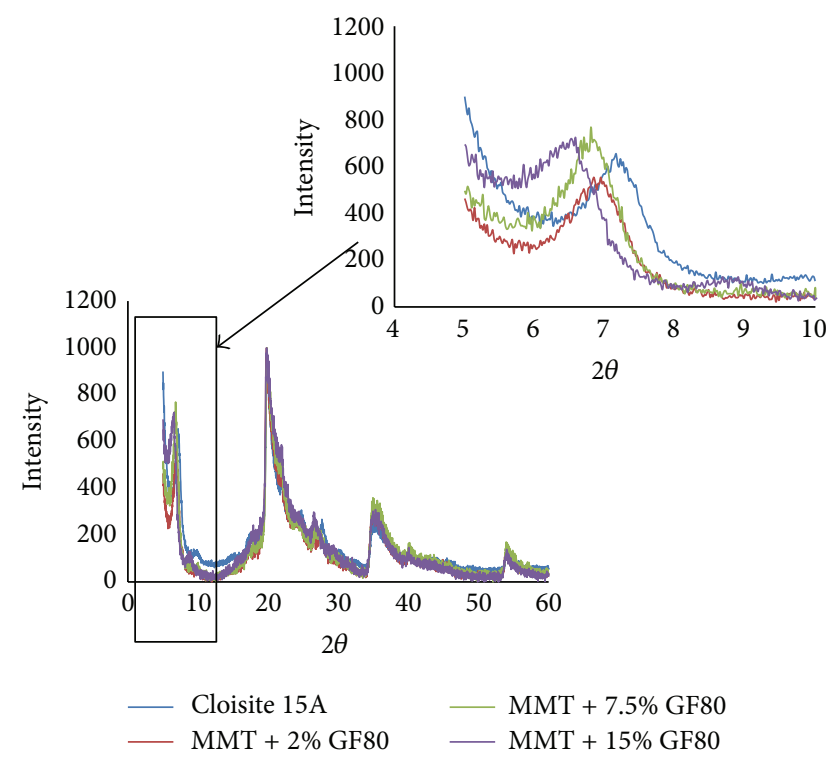

FIGURE 2: WAXS diffractograms of unmodified MMT (red), MMT with $2 \%$ w/w GF80 (blue), MMT with 7.5\% w/w GF80 (green), and MMT with 15\% w/w GF80 (violet).

According to Bragg's law (equation (1)), lower $2 \theta$ indicates an increase in the distance of the diffraction planes, that is, an increase in the basal spacing. Neat MMT presents a single basal peak at $7.19^{\circ}(2 \theta)$, corresponding to a $d_{001}$-spacing of $12.28 \AA$, which is the basal space distance of the natural clay [30]. The patterns of MMT samples modified with different amounts of silane show basal reflections at $6.87^{\circ}\left(d_{001}\right.$-spacing $=12.86 \AA)$ in the case of MMT $-2 \%$ and MMT $-7.5 \%$ and at $6.47^{\circ}\left(d_{001}\right.$-spacing $\left.=13.65 \AA\right)$ for MMT $-15 \%$, indicating an increase in the distance of the diffraction planes of $0.58 \AA$ and $1.37 \AA$, respectively. This phenomenon reveals that some intergallery expansion has taken place thanks to the presence of silane and the fact that the higher the quantity of silane the higher the spacing between lamellae.

WAXS analyses were used also to determine the crystalline structure of nanocomposite PLAs: the analysis was performed on the films obtained from casting as described in Section 2.1. WAXS patterns of MMT, of STD PLA, and of PLA containing $1.0 \% \mathrm{w} / \mathrm{w}$ and $0.1 \% \mathrm{w} / \mathrm{w}$ of unmodified MMT (PLA1 and PLA3, resp.), collected in the range of $2 \theta=5-60^{\circ}$, are presented in Figure 3. In PLA1 and PLA3 a diffraction peak is present, at $2 \theta=16.82^{\circ}$ and $16.94^{\circ}$, respectively,

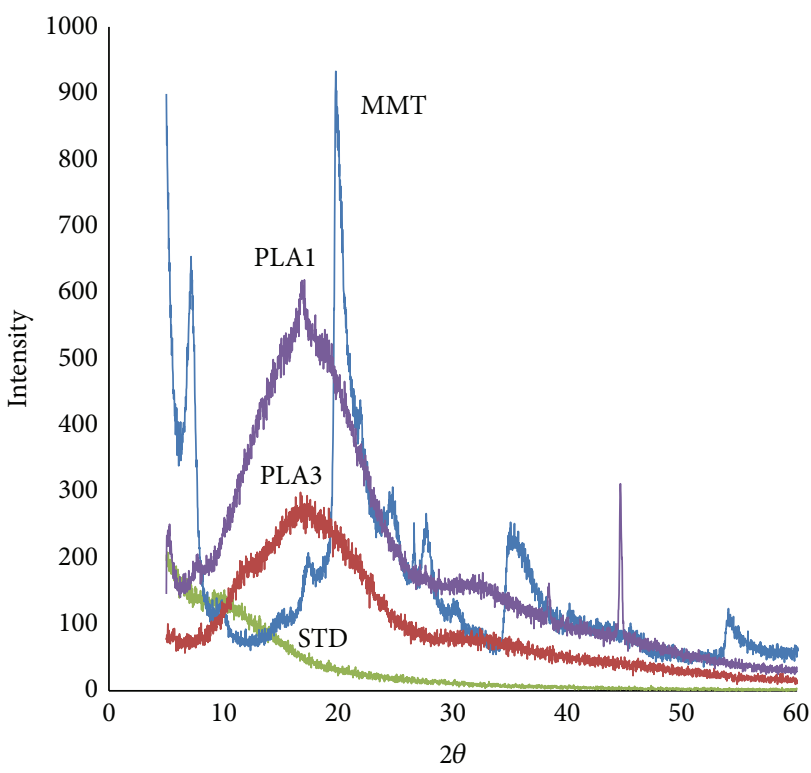

FIgURE 3: WAXS diffractograms of Cloisite 15A powder, STD PLA, PLA1, and PLA3.

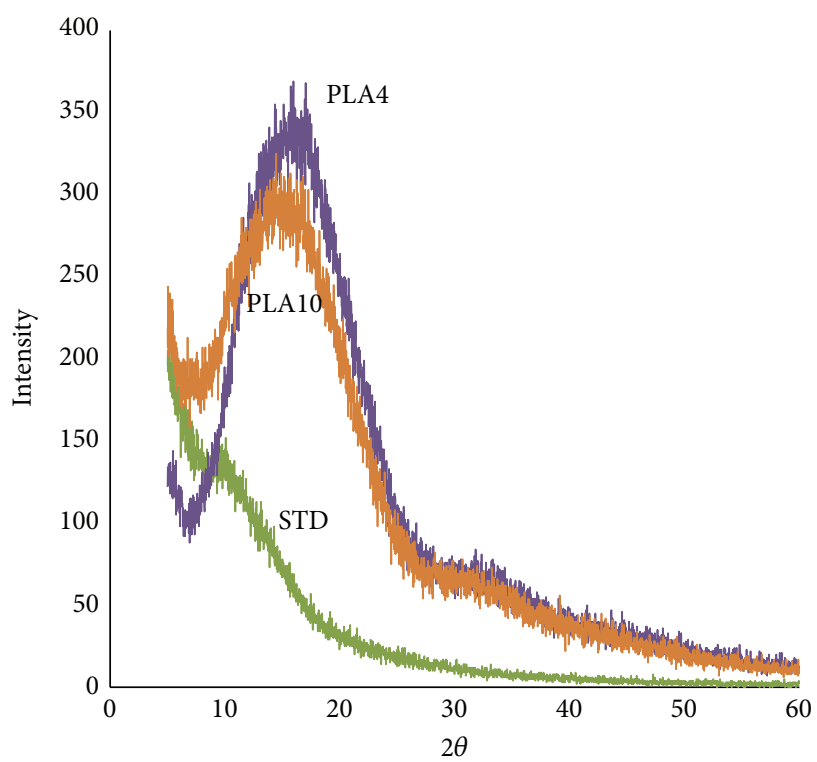

FIgURE 4: WAXS diffractograms of STD PLA, PLA4, and PLA10.

consistent with the major crystalline peak of highly crystalline PLA [31].

It is possible to observe an increase of the height of the diffraction peak as the amount of MMT increases. This behavior indicates that MMT promotes heteronucleation process.

Figure 4 shows WAXS patterns of STD PLA and of PLA with $1 \%$ w/w of modified MMT with $2 \%$ w/w of GF80 (PLA4) and with 15\% w/w of GF80 (PLA10). The nanocomposites patterns show that diffraction peaks of PLA4 and PLA10 are higher than STD; this behavior indicates that modified MMT promotes the intercalation of MMT in PLA chains. 


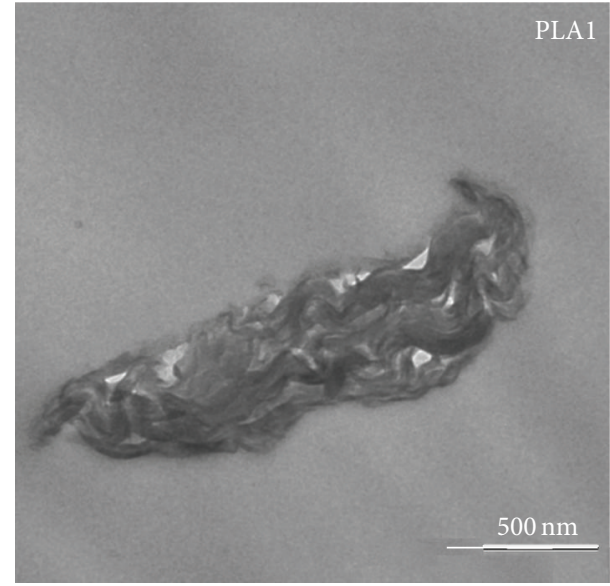

(a)

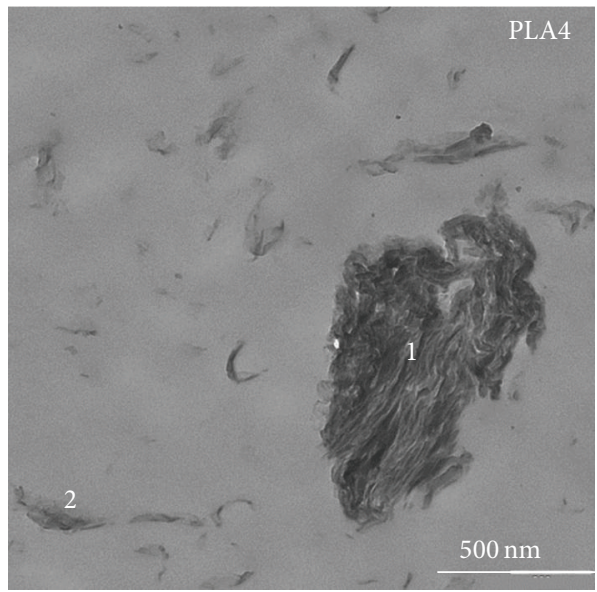

(c)

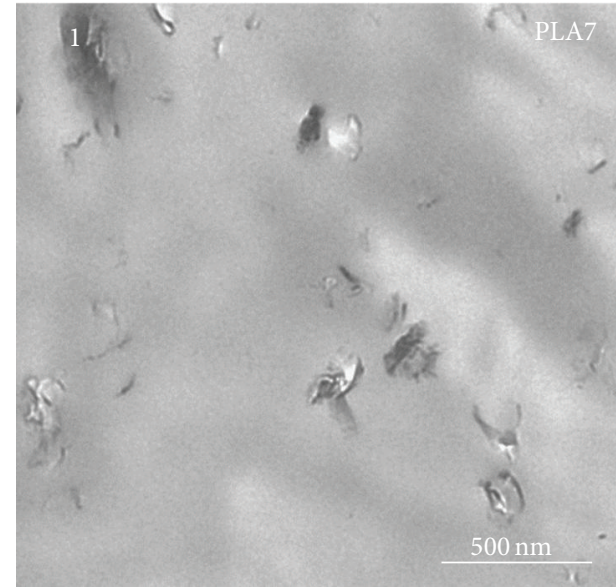

(b)

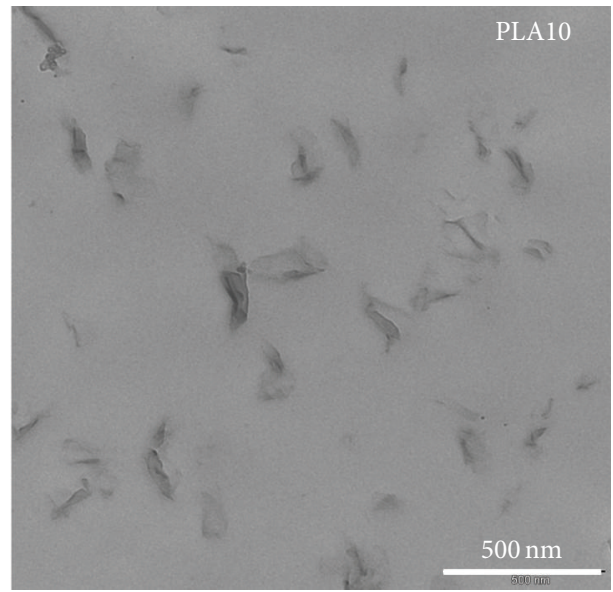

(d)

Figure 5: TEM images 1\% w/w of PLA1 (a), PLA7 (b), PLA4 (c), and PLA10 (d).

3.2. TEM Images. Figure 5 reports TEM images of PLA nanocomposites; in PLA1, unmodified MMT is dispersed in the polymeric matrix, with aggregates having big dimensions indicating that the mineral has not been efficiently exfoliated. A good exfoliation should result in a good dispersion of MMT platelets within the polymeric matrix, each platelet having a length of about 100-200 nm, a similar width, and a thickness of some nanometers [32,33]. In Figure 5, the effect of silane is visible: as its quantity increases, the dispersion of MMT platelets is enhanced. In PLA1 an aggregate is shown having big dimensions (maximum length $=1.6 \mu \mathrm{m}$, width $500 \mathrm{~nm}$ ): similar aggregates are scattered through the nanocomposite. In PLA4, aggregates are present together with better exfoliated platelets: the bigger aggregate (number 1) has a length of about $1 \mu \mathrm{m}$ and width of about $500 \mathrm{~nm}$. Other big aggregates (number 2) are about $500 \mathrm{~nm} \times 100 \mathrm{~nm}$ or less: other particles have an average length of $154 \pm 100 \mathrm{~nm}$ and an average width of $41 \pm 25 \mathrm{~nm}$ (calculated on 20 particles). In PLA7 aggregates with lower dimension are visible, with the bigger one (number 1) being about $500 \mathrm{~nm} \times 130 \mathrm{~nm}$ : average length is $122 \pm 55 \mathrm{~nm}$ and width is $38 \pm 18 \mathrm{~nm}$. In PLA4 and PLA7 the presence of some aggregates influences the dispersity of data collected. In Supporting Information, images of PLA4 (see S1 in Supplementary Material available online at http://dx.doi.org/10.1155/2015/418418) and PLA7 (S2) with lower magnification are presented. In PLA10, MMT is homogeneously dispersed and no big aggregates are visible: average length is $110 \pm 40 \mathrm{~nm}$; average width is $33 \pm 15 \mathrm{~nm}$.

3.3. Size Exclusion Chromatography (SEC). Numeral average molecular weights $(\overline{M n})$ and molecular weight distribution $(D)$ determined via SEC and expressed as polystyrene equivalents are provided in Table 5; as described in Section 2.1, samples were filtered with a $0.45 \mu \mathrm{m}$ filter; therefore molecular weight data refer only to the polymer present in the solution.

Samples PLA1, PLA2, and PLA3, containing unmodified MMT, show that the presence of pure MMT interferes with the polymerization process, lowering the molecular weights as the quantity of mineral increases. Two possible explanations for this phenomenon can be given. The first one is related to the fact that $-\mathrm{OH}$ groups on the surface of unmodified MMT can potentially initiate the polymerization, as already reported in literature [34]. Since molecular weight 
TABLE 5: SEC data of PLAs synthesized.

\begin{tabular}{lcc}
\hline Sample & $\overline{M n}$ & $D$ \\
\hline STD & 54124 & 1.79 \\
PLA1 & 29901 & 1.26 \\
PLA2 & 28563 & 1.33 \\
PLA3 & 33238 & 1.29 \\
PLA4 & 59230 & 1.86 \\
PLA5 & 57431 & 1.85 \\
PLA6 & 53611 & 1.80 \\
PLA7 & 61551 & 1.88 \\
PLA8 & 62045 & 1.89 \\
PLA9 & 62117 & 1.89 \\
PLA10 & 59683 & 1.88 \\
PLA11 & 58661 & 1.87 \\
PLA12 & 59342 & 1.89 \\
PLA13 & 53991 & 1.77 \\
\hline
\end{tabular}

distribution values of PLA1, PLA2, and PLA3 are much lower than that of STD, a second possible explanation, alternative to the first one, is related to the fact that the free acidic sites of unmodified MMT may slow the kinetics of polymerization process partially deactivating the catalyst. The lower activity of catalyst decreases the kinetics of ROP but also the kinetics of transesterification reaction that is the cause of polydispersity increase.

PLA13, containing a very high quantity of catalyst (3\% $\mathrm{w} / \mathrm{w})$ and of MMT $(5 \% \mathrm{w} / \mathrm{w})$, was synthesised to confirm the validity of one of these hypotheses: SEC analysis shows that $\overline{M n}$ and $D$ of PLA13 are very close to those of STD sample $(\overline{M n}: 53991 ; D: 1.77)$. These values demonstrate that pure MMT has the tendency to deactivate part of the catalyst, since higher amounts of tin octanoate lead to a PLA with high molecular weight; the second hypothesis is therefore confirmed.

Nanocomposites containing silane-modified MMT have $\overline{M n}$ comparable with that of pure PLAs even when only $2 \%$ $\mathrm{w} / \mathrm{w}$ of silane is used. Molecular weights tend to increase as the quantity of silane increases, indicating that epoxy silane reacting with MMT -OH surface groups decreases the interaction of MMT with catalyst.

Since SEC refers only to the polymer that is present in solution, two samples were synthesized to confirm the reaction between epoxy silane and end groups of the growing polymeric chain: one using the same feed and conditions of PLA3 (named PLA14) and one using the same feed and conditions of PLA12 (named PLA15); in both PLA14 and PLA15 a tetrafunctional comonomer, pyromellitic dianhydride (PMDA, $3.56 * 10^{-5} \mathrm{~mol}$ in $50 \mathrm{~g}$ of lactide), was added in the feed. In PLA15, 0.1\% w/w on lactide of MMT having $712 \mathrm{meq} / \mathrm{kg}$ of silane (i.e., $712 \mathrm{meq} / \mathrm{kg}$ of epoxy groups, see Table 1) is introduced in the feed. This means that the concentration of epoxy groups deriving from silane-modified MMT is $0.712 \mathrm{meq} / \mathrm{kg}$ of lactide (i.e., $3.56 * 10^{-5} \mathrm{~mol}$ in $50 \mathrm{~g}$ of lactide). The concentration of PMDA used is therefore equal to the concentration of epoxy groups present in the feed of
PLA15 through MMT. On the contrary PLA14 has no epoxy groups deriving from MMT.

During the bulk polymerization of lactide, PMDA behaves as a tetrafunctional acid comonomer, because anhydride moieties react after nucleophilic attack of $-\mathrm{OH}$ groups of PLA growing chains and the other two carboxyl functions, which arise after the ring opening of anhydride, can react during the SSP process, where operating conditions allow shifting the equilibrium of the reaction, with $-\mathrm{OH}$ groups deriving either by subsequent opening of epoxide groups of the silane (PLA15) or by $-\mathrm{OH}$ end groups of PLA chains, as reported in Figures 6 and 7; out of clarity, in these two figures only two chains growing from PMDA are shown: other chains behave in the same way.

The presence of a multifunctional reagent such as PMDA should result in two different behaviours of PLA14 and PLA15: PLA14, having no silane, has a marked shear thinning behavior, but it is anyway possible to determine its zero shear viscosity $\left(\eta_{0}=1.88 * 10^{4} \mathrm{~Pa} * \mathrm{~s}\right)$. The sample is soluble in $\mathrm{CH}_{2} \mathrm{Cl}_{2}$, indicating that no cross-linking has occurred, but only partial branching of the macromolecular chains. PLA15, on the contrary, is not soluble in methylene chloride and melt viscosity has no plateau at low shear rates: this behavior is typical of highly branched and/or partially crosslinked polymers (Figure 8). This clearly indicates that there has been a reaction between the epoxy functions of organosilane with $-\mathrm{OH}$ groups of the growing chains.

3.4. Thermal Analyses (DSC). Figure 9 reports DSC thermogram of STD PLA compared to PLA1, PLA2, and PLA3 having, respectively, $1-0.5-0.1 \% \mathrm{w} / \mathrm{w}$ of MMT. STD sample crystallizes on heating (cold crystallization) giving a crystalline phase that is characterized by two melting peaks. It has already been reported that the double melting behaviour can be linked to the formation of two different crystal forms: the $\alpha$-one-pseudo-orthorhombic, pseudo-hexagonal, or orthorhombic (melting at higher temperature) and the $\beta$-one-orthorhombic or trigonal [35]. The most common polymorph is the $\alpha$-form while the packing of $\beta$-form has been interpreted as an imperfect crystal structure. The helical conformations of the chains in the $\alpha$ - and $\beta$-structures have approximately the same energy, and thus their assembling must be the main reason for the existence of two different crystal structures [36].

On the other hand, it has been reported that the multiple melting behaviour in PLA could be also linked to a crystal reorganization upon melting, a fraction of imperfect crystals formed in melt crystallization which would have a high tendency to reorganize into more ordered structures and melt at higher temperature [36]. In this work, melt crystallization of STD is negligible, suggesting that the double melting behaviour observed cannot be attributed to a melt crystallization phenomenon but rather to the presence of both $\alpha$-form, which is predominant, and $\beta$-form.

Table 6 summarizes thermal data for STD PLA and PLA/MMT nanocomposites with no silane. In the table $\chi_{\mathrm{c}}$ indicates the crystalline fraction of the samples that was evaluated using (2); transition temperatures are taken according to Figure $1 . T_{\mathrm{g}}$ is glass transition temperature, $\Delta C_{p}$ is 


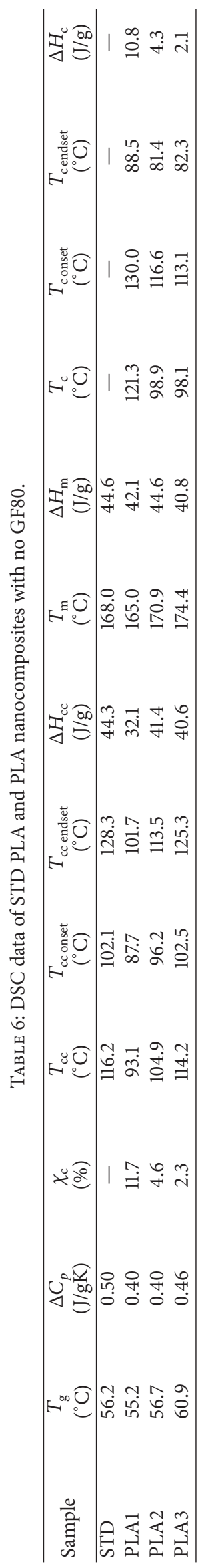




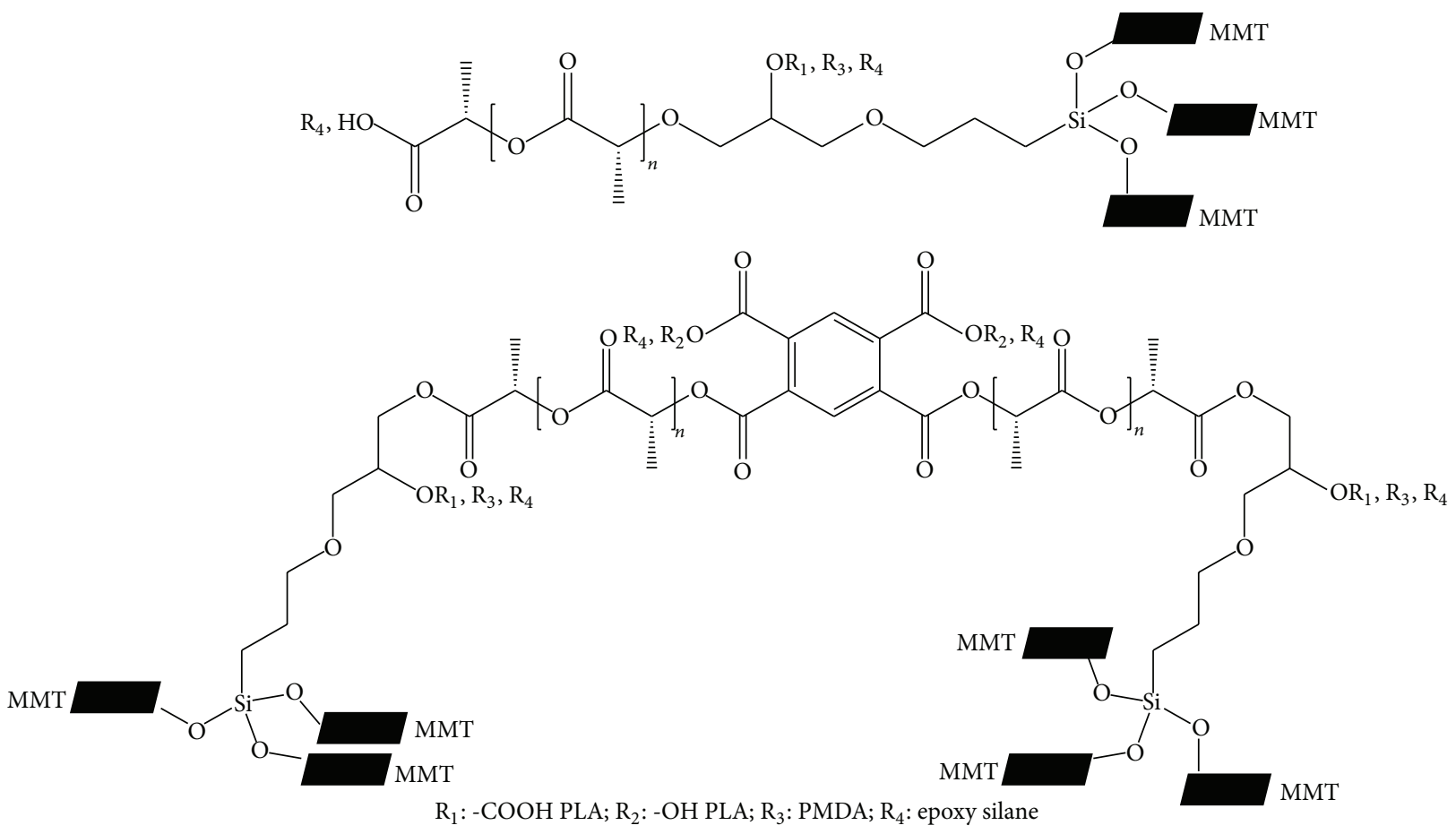

FIgURE 6: Possible structures of PLA nanocomposites branched with PMDA containing silane-modified MMT chemically linked to the polymer.

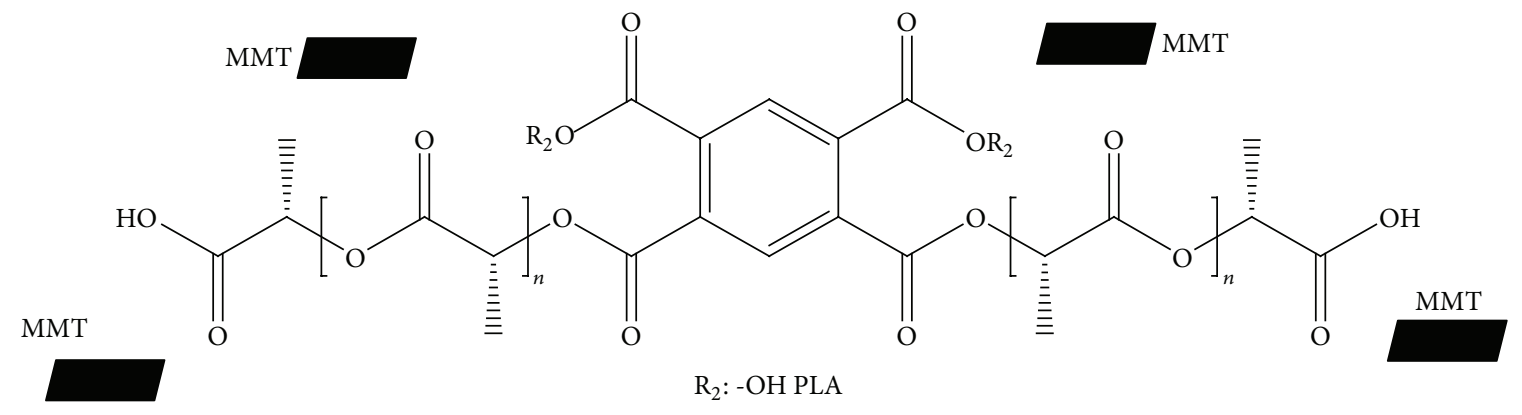

FIGURE 7: Structure of PLA nanocomposites branched with PMDA containing unmodified MMT.

the specific heat, $T_{\mathrm{cc}}$ is the cold crystallization temperature, $\Delta H_{\mathrm{cc}}$ is the cold crystallization enthalpy, $T_{\mathrm{m}}$ is melting temperature, $\Delta H_{\mathrm{m}}$ is melting enthalpy, $T_{\mathrm{c}}$ is melt crystallization temperature, and $\Delta H_{\mathrm{c}}$ is melt crystallization enthalpy as indicated in Materials and Methods.

3.4.1. PLA1-PLA3: $T_{c}$ and $\chi_{c} . T_{c}$ is absent in STD; unmodified MMT enhances the crystallization process and increases $\chi_{c}$ as the content of MMT increases: a small melt crystallization peak becomes visible (Figure 9). $T_{\mathrm{c}}$ increases significantly as the quantity of MMT increases, going from $98.1^{\circ} \mathrm{C}$ (PLA3) to $121.3^{\circ} \mathrm{C}$ (PLA1) with a difference of $23.2^{\circ} \mathrm{C} ; \Delta H_{\mathrm{c}}$ is low in any case. As a conclusion, MMT promotes crystalline features of the nanocomposites, acting as heterogeneous crystalline promoter.

3.4.2. PLA1-PLA3: $T_{g}$ and $\triangle C_{p}$. The effect of different layered silicate nanoparticles on $T_{\mathrm{g}}$ in polymer nanocomposites has been studied in several works where PLA nanocomposites are obtained via extrusion but results seem controversial: in some cases, $T_{\mathrm{g}}$ increases have been reported [37]; in others a decrease has been demonstrated [38] or no relevant influence has been observed [39]. In the present paper, $T_{\mathrm{g}}$ of nanocomposites lowers as the quantity of MMT increases: in PLA3 glass transition is $4.7^{\circ} \mathrm{C}$ higher than in STD but in PLA1 it becomes $1.0^{\circ} \mathrm{C}$ lower than in STD.

Higher MMT quantities tend also to decrease $\Delta C_{p}$ : with low amounts of MMT, as in PLA3, $\Delta C_{p}$ is close to STD getting lower for PLA2 and PLA1. As expected, higher $\chi_{c}$ causes lower $\Delta C_{p}$ of the glass transition.

3.4.3. PLA1-PLA3: $T_{c c}$ and $T_{m}$. $T_{c c}$ lowers as MMT content increases: in PLA3 $T_{c c}$ and $T_{c c}$ onset and endset are similar to STD whereas for PLAl there is a decrease of $T_{\mathrm{cc}}$ of $23.1^{\circ} \mathrm{C}$ in comparison to STD. The presence of MMT results also in lower $T_{\mathrm{m}}$ as MMT content increases in comparison with 


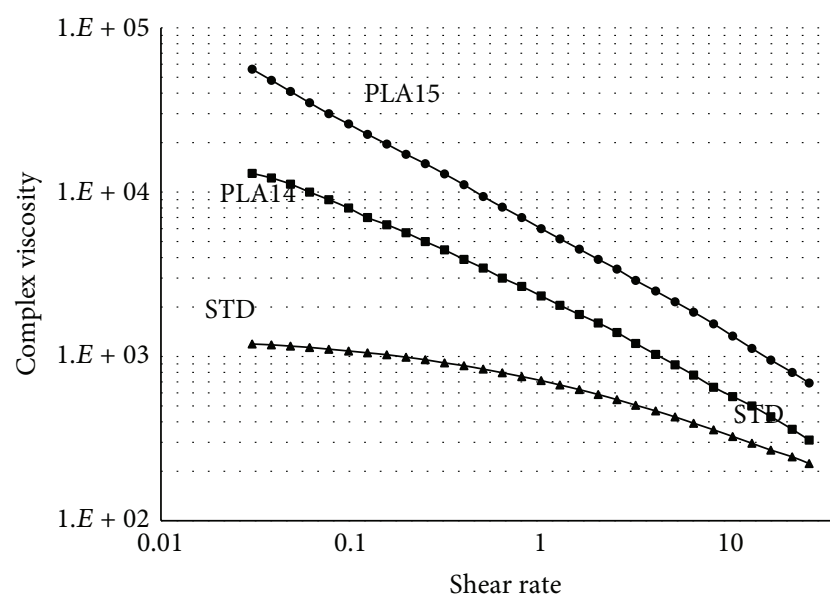

FIgURE 8: Rheological curves of PLA nanocomposites with PMDA compared with STD sample.

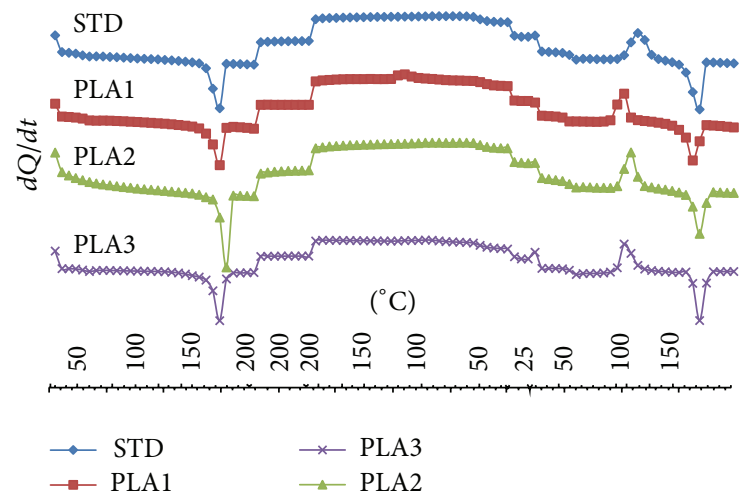

FigURE 9: DSC thermograms of PLA and nanocomposites.

STD. These phenomena are reasonably due to a different crystallization process caused by the presence of MMT tallies; analogous trends have been already observed in a previous work [40].

Thermal data of PLA4-PLA12, containing silanemodified MMT, are shown in Table 7. DSC thermograms of PLA4, PLA7, and PLA10 and PLA6, PLA9, and PLA12 are reported in Supporting Information (S3 and S4).

3.4.4. PLA4-PLA12: $T_{c}$ and $\chi_{c}$. In all nanocomposites, melt crystallization peak is visible with an increase of $\chi_{\mathrm{c}}$ content with respect to STD sample: low quantities of MMT allow for high $T_{c}$ that lowers as the quantity of MMT increases. This trend is opposite to that of PLA1, PLA2, and PLA3. This is probably due to the enhanced dispersion of silane modified MMT that increases surface area of the mineral making it a very effective heterogeneous crystallization promoter.

3.4.5. PLA4-PLA12: $T_{g}$ and $\triangle C_{p}$. When silane is present, low amounts of MMT, namely, PLA6, PLA9, and PLA12, do not increase glass transition in comparison to STD as happening for PLA3. Increasing the quantity of MMT, $T_{\mathrm{g}}$ increases but

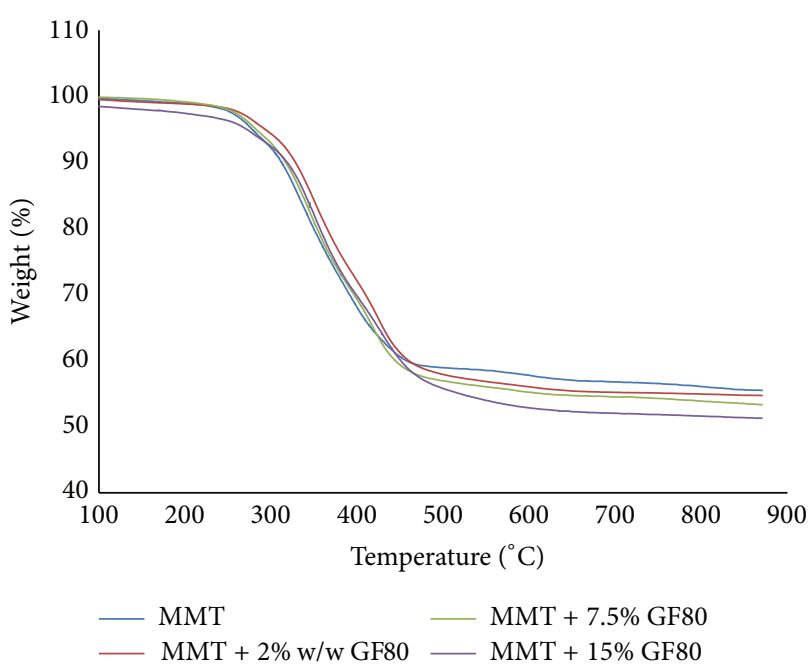

Figure 10: TGA curves of Cloisite 15A modified with different amounts of GF80.

not significantly. In all samples, $\Delta C_{p}$ is lower than that of STD as already seen for PLA1-PLA3.

3.4.6. PLA4-PLA12: $T_{c c}$ and $T_{m}$. $T_{c c}$ in all samples of Table 7 is lower than in STD; when no silane is present (PLA1-PLA2PLA3), the lower the quantity of MMT, the higher the $T_{\mathrm{cc}}$. When silane is present on the surface of MMT, the opposite trend is seen: $T_{\mathrm{cc}}$ is higher with $0.5 \%$ and $1 \%$ of MMT. No real trend can be observed regarding melt behaviour: in general, it seems that, for samples with the same quantity of silane, $T_{\mathrm{m}}$ tends to decrease as MMT content decreases.

As a general consideration, in all nanocomposite samples a melt crystallization peak, that is absent in STD, is visible; both $T_{\mathrm{c}}$ and $T_{\mathrm{cc}}$ are significantly affected by the presence of silane, showing that very small quantities of modified MMT promote these processes.

Silane, enhancing the dispersion of MMT, contributes to making it a very effective heterogeneous crystallization promoter, slightly increasing crystalline fraction of the nanocomposites.

3.5. Thermogravimetric Analyses (TGA). Thermogravimetric analyses were performed both on MMT and on PLA nanocomposites. TGA was performed on MMT to check the validity of the results obtained via potentiometric titration of the silane; PLA nanocomposites were analysed to evaluate their resistance to thermal degradation.

3.5.1. TGA on MMTs. Analyses were performed on pure and on silane-modified MMTs (Figure 10).

Weight loss is directly proportional to the quantity of silane present on MMT, in accordance with potentiometric titration results (Table 8 and Figure 10).

In Figure 11 a correlation between amount of silane determined via potentiometric titration and weight loss is reported; variance value $\left(R^{2}=0.9991\right)$ suggests that titration and TGA data are in very good agreement with each other. 


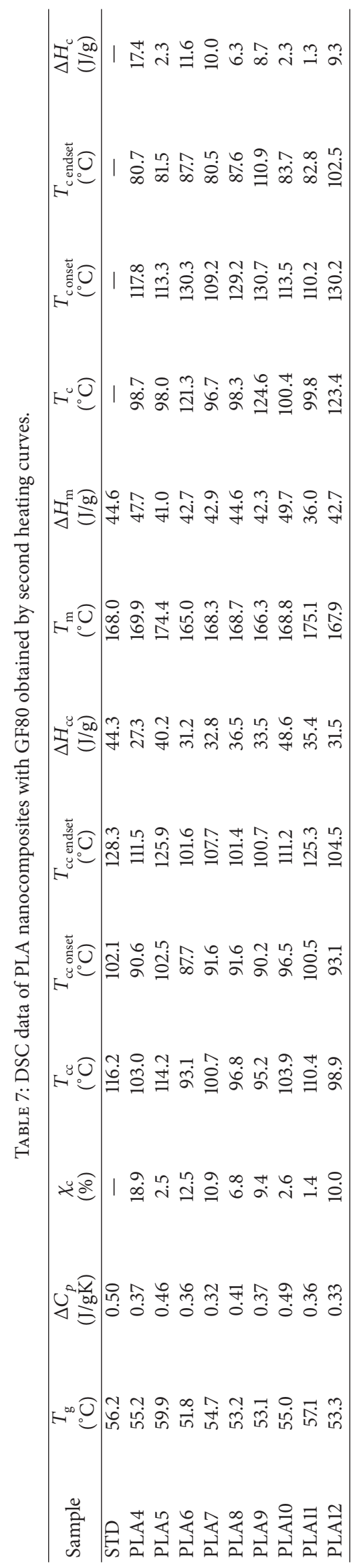


TABle 8: Amount of silane present on Cloisite 15A surface and its weight loss.

\begin{tabular}{|c|c|c|c|c|}
\hline $\begin{array}{l}\text { Silane added } \\
\text { (w/w \% on MMT) }\end{array}$ & $\begin{array}{c}\mathrm{meq} / \mathrm{kg} \\
\text { (titration) }\end{array}$ & $\begin{array}{l}\text { Weight loss (\%) } \\
\text { at } 900^{\circ} \mathrm{C}\end{array}$ & $\begin{array}{l}\Delta \text { Weight loss (\%) } \\
\text { with respect to } \\
\text { unmodified MMT }\end{array}$ & $\begin{array}{c}\text { MW of the residue } \\
(\mathrm{g} / \mathrm{mol})\end{array}$ \\
\hline Cloisite 15A & - & 44.49 & - & - \\
\hline $2 \%$ GF 80 & 119 & 45.22 & 0.73 & 61.34 \\
\hline $7.5 \%$ GF80 & 380 & 46.67 & 2.18 & 57.37 \\
\hline $15 \%$ GF80 & 712 & 48.72 & 4.23 & 59.41 \\
\hline
\end{tabular}

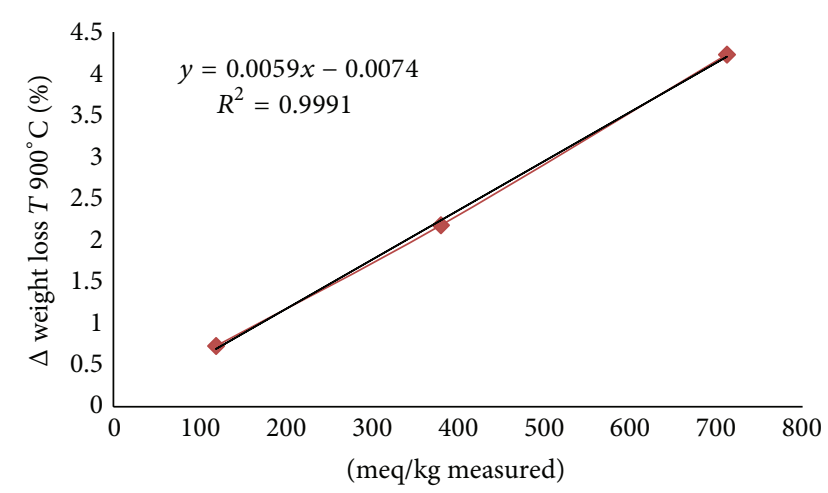

FIGURE 11: Correlation between amount and silane weight loss on Cloisite 15A surface.

Thanks to the combined use of titration and TGA, it is possible to determine the molecular weight of TGA residues calculated from $\Delta$ weight loss (\%) with respect to unmodified MMT, which are in the range between 57.37 and $61.34 \mathrm{~g} / \mathrm{mol}$; these values suggest that only the inorganic part of the silane, represented by $\mathrm{SiO}_{2}(60.08 \mathrm{~g} / \mathrm{mol})$, remains when reaching high temperatures.

3.5.2. TGA on PLA Nanocomposites. Many authors considered several molecular as well as radical mechanisms to explain PLA thermal degradation: according to McNeill and Leiper [41], the main reaction route is a nonradical, backbiting ester interchange reaction involving $-\mathrm{OH}$ chain ends. This reaction mechanism can, depending upon the size of the cyclic transition state, produce oligomers or acetaldehyde plus carbon monoxide.

Kopinke et al. [42] also propose a radical reaction which starts either with an alkyl-oxygen or with an acyl-oxygen hemolysis involving the formation of several types of oxygenand carbon-centered macroradicals plus carbon monoxide.

Besides, several works $[43,44]$ reported that the addition of layered silicate nanoparticles, such as MMT, to PLA can influence thermal properties, mainly thermal degradation, in comparison to the unfilled matrix and that the presence of organic ions between the lamellae, especially at higher clay content, accelerates PLA degradation process, probably due to catalysis by the hydroxyl groups belonging to the silicate layers surface and by the organic modifier [45].

Thermal stabilities of STD, PLA1, PLA2, and PLA3 were estimated evaluating temperatures corresponding to $1 \%, 10 \%$, $50 \%$, and $95 \%$ of weight loss $\left(T_{1 \%}, T_{10 \%}, T_{50 \%}\right.$, and $\left.T_{95 \%}\right)$;
TABle 9: Degradation data of the samples synthesized.

\begin{tabular}{lcccc}
\hline Sample & $T_{1 \%}\left({ }^{\circ} \mathrm{C}\right)$ & $T_{10 \%}\left({ }^{\circ} \mathrm{C}\right)$ & $T_{50 \%}\left({ }^{\circ} \mathrm{C}\right)$ & $T_{95 \%}\left({ }^{\circ} \mathrm{C}\right)$ \\
\hline STD & 275.2 & 319.4 & 359.2 & 384.7 \\
PLA1 & 257.0 & 296.0 & 320.8 & 337.8 \\
PLA2 & 249.1 & 287.6 & 311.6 & 329.3 \\
PLA3 & 268.8 & 295.5 & 320.2 & 338.4 \\
\hline
\end{tabular}

results obtained are reported in Table 9. TGA curves of STD and PLA1, PLA2, and PLA3 are presented in Supporting Information (S5).

Thermal stability in comparison to STD decreases: both the beginning of degradation $\left(T_{1 \%}\right)$ and the end $\left(T_{95 \%}\right)$ occur at lower temperature. The decrease of thermal stability probably is due to both the presence of more $-\mathrm{OH}$ chain ends in samples with lower molecular weights in comparison to STD and the interaction with surface $-\mathrm{OH}$ of unmodified MMT.

Thermal data of PLA nanocomposites containing silanemodified MMT are reported in Table 10. TGA curves of PLA4-12 are shown in Supporting Information (S6 and S7).

$T_{1 \%}$ of PLA8 $\left(7.5 \%\right.$ w/w silane/MMT) is $45.9^{\circ} \mathrm{C}$ higher than that of STD and $T_{10 \%}$ is still very high $\left(42.3^{\circ} \mathrm{C}\right.$ higher than STD); PLA8, PLA9, PLA10, PLA11, and PLA12 show marked increases of thermal stability in the range of $T_{1 \%}-T_{95 \%}$ in comparison to STD. The higher degradation temperature in comparison with PLA1, PLA2, and PLA3 can be explained considering that there are less $-\mathrm{OH}$ chain ends able to be involved in the backbiting ester interchange reaction and that there is no interaction of surface $-\mathrm{OH}$ from modified MMT due to the presence of organosilane.

As a general statement, all samples with modified MMT, except for PLA4 and PLA7, show higher thermal stability in comparison to STD: $T_{1 \%}$ and $T_{10 \%}$ increase as silane content increases, indicating that thermal stability is promoted by the silane. The lower thermal stability of PLA4 and PLA7 can be explained considering that these samples have high MMT content $(1 \% \mathrm{w} / \mathrm{w})$ and relatively low amounts of silane on the surface. This implies that MMT platelets still have $-\mathrm{OH}$ moieties on the surface promoting degradation and at the same time, being better dispersed than PLA1, a high surface area in contact with the polymeric matrix. The combination of these two factors, $-\mathrm{OH}$ groups available on the surface and high surface area in contact with the polymer, determines lower thermal stability in comparison to both STD and other modified PLA nanocomposites. 
TABLE 10: Degradation data of the samples synthesized.

\begin{tabular}{lllll}
\hline Sample & $\begin{array}{l}T_{1 \%} \\
\left({ }^{\circ} \mathrm{C}\right)\end{array}$ & $\begin{array}{l}T_{10 \%} \\
\left({ }^{\circ} \mathrm{C}\right)\end{array}$ & $\begin{array}{c}T_{50 \%} \\
\left({ }^{\circ} \mathrm{C}\right)\end{array}$ & $\begin{array}{c}T_{95 \%} \\
\left({ }^{\circ} \mathrm{C}\right)\end{array}$ \\
\hline STD & 275.2 & 319.4 & 359.2 & 384.7 \\
PLA4 & 245.4 & 277.1 & 304.6 & 321.1 \\
PLA5 & 289.5 & 339.8 & 382.8 & 400.4 \\
PLA6 & 300.1 & 350.4 & 386.1 & 403.0 \\
PLA7 & 243.5 & 279.2 & 309.6 & 327.0 \\
PLA8 & 321.1 & 361.7 & 386.0 & 400.3 \\
PLA9 & 323.9 & 348.8 & 375.0 & 400.0 \\
PLA10 & 299.0 & 346.5 & 364.5 & 382.8 \\
PLA11 & 303.2 & 325.4 & 367.0 & 386.0 \\
PLA12 & 324.6 & 351.6 & 368.9 & 386.3 \\
\hline
\end{tabular}

TABLE 11: Zero shear viscosity of PLA nanocomposites.

\begin{tabular}{lc}
\hline Sample & $\eta_{0}(\mathrm{~Pa} * \mathrm{~s})$ \\
\hline STD & $1.19 * 10^{3}$ \\
PLA1 & $5.96 * 10^{3}$ \\
PLA2 & $4.38 * 10^{3}$ \\
PLA3 & $3.31 * 10^{3}$ \\
PLA4 & $8.42 * 10^{3}$ \\
PLA5 & $7.63 * 10^{3}$ \\
PLA6 & $5.96 * 10^{3}$ \\
PLA7 & $4.5 * 10^{3}$ \\
PLA8 & $4.3 * 10^{3}$ \\
PLA9 & $3.6 * 10^{3}$ \\
PLA10 & $68.2 * 10^{3}$ \\
PLA11 & $62.3 * 10^{3}$ \\
PLA12 & $56.7 * 10^{3}$ \\
\hline
\end{tabular}

3.6. Rheological Analyses. Rheological curves obtained via frequency sweep experiments on STD, PLA1, PLA2, and PLA3 are presented in Figure 12.

Curves have viscoelastic behaviour, with a Newtonian plateau at low shear rates and shear thinning behaviour. Zero shear viscosity values, $\eta_{0}$ (shown in Table 11 ), have been calculated using Cross equation (3) passing from experimental data obtained as complex viscosity versus angular frequency to data as standard shear viscosity versus shear rate thanks to Cox-Merz rule.

In Cross equation, $\eta_{0}$ represents zero shear viscosity, $\eta_{\infty}$ is infinite-shear viscosity, $\dot{\gamma}$ is shear rate, $(1-n)$ is known as Cross rate constant ( $n$ is non-Newtonian behaviour index), it is dimensionless and is a measure of the degree of dependence of viscosity on shear rate in the shear thinning region, and $K$ is known as the Cross time constant:

$$
\frac{\eta-\eta_{\infty}}{\eta_{0}-\eta_{\infty}}=\frac{1}{1+\left(K^{2} \cdot \dot{\gamma}^{2}\right)^{(1-n) / 2}}=\frac{1}{1+(K \cdot \dot{\gamma})^{1-n}} .
$$

Figure 12 shows that the presence of MMT consistently increases the viscosity of the melt even if SEC shows that the soluble part of PLA1-PLA3 has molecular weights far lower

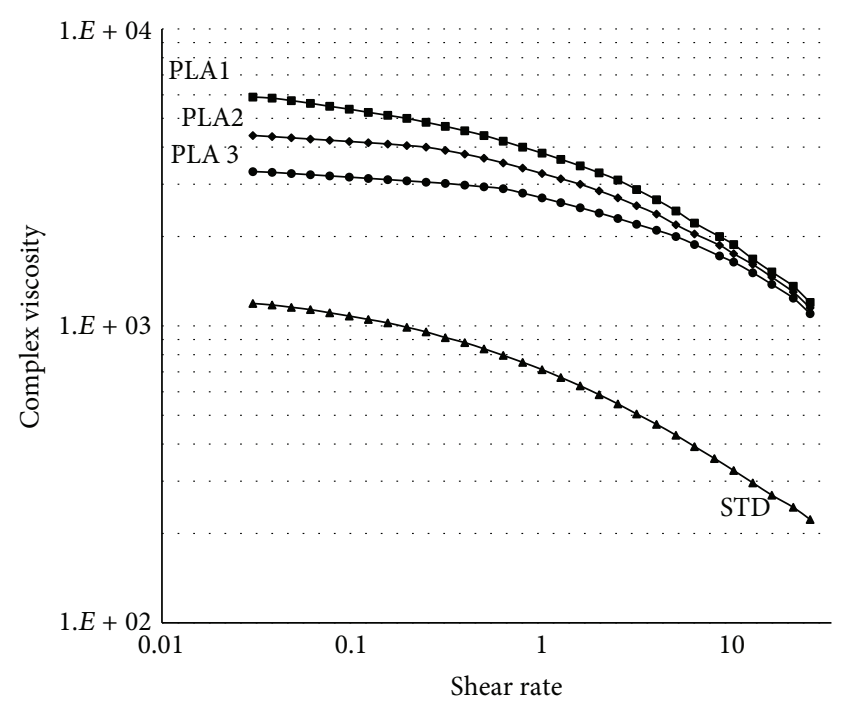

Figure 12: Rheological curves of STD and PLA1, PLA2, and PLA3.

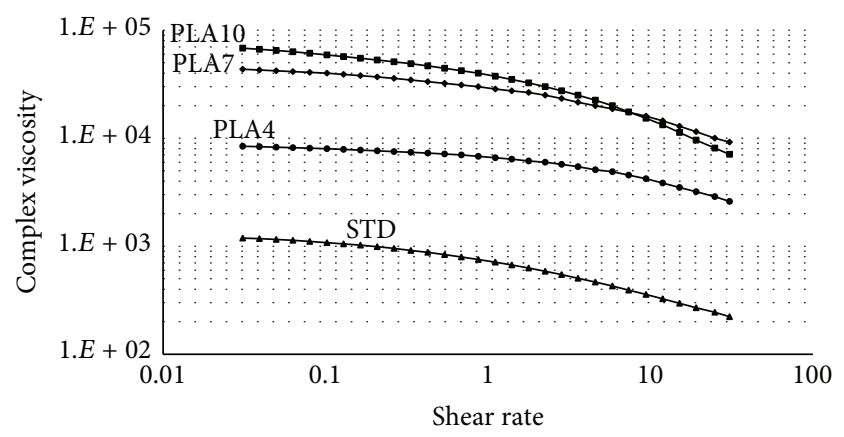

FIGURE 13: Rheological curves of STD PLA and modified samples.

than those of STD. $\eta_{0}$ is much higher and increases considerably as MMT content increases.

Bearing in mind that SEC data refer only to the polymer dissolved, and therefore only to the polymer that is not chemically bonded to the nanoparticles, the high values of $\eta_{0}$ of unmodified samples can be due to $-\mathrm{C}=\mathrm{O}$ groups of PLA that alter the normal flow of STD PLA, flowing with higher difficulty in the direction of flow and promoting the formation of a percolating network derived from the reciprocity among the strong relating sheet particles as a result of the preferential orientation of MMT layers [46].

It is important to underline that, thanks to in situ synthesis, the effects of MMT on melt viscosity are greatly enhanced. Wang et al. [47] studying melt compounding of commercial PLA and MMT reported that far higher quantities of MMT $(>5 \% \mathrm{w} / \mathrm{w})$ were required to reach viscosity values similar to those of neat PLA and also that the inclusion of small amounts of MMT (1\% w/w) lowers the complex viscosity of PLA matrix. Using "in situ" synthesis, even $0.1 \% \mathrm{w} / \mathrm{w}$ of MMT allows obtaining melt viscosity values far higher than those of neat PLA. When silane is present on the surface of MMT (Figure 13), the effect on melt viscosity is magnified; melt viscosity at low shears further increases, and the shear thinning 

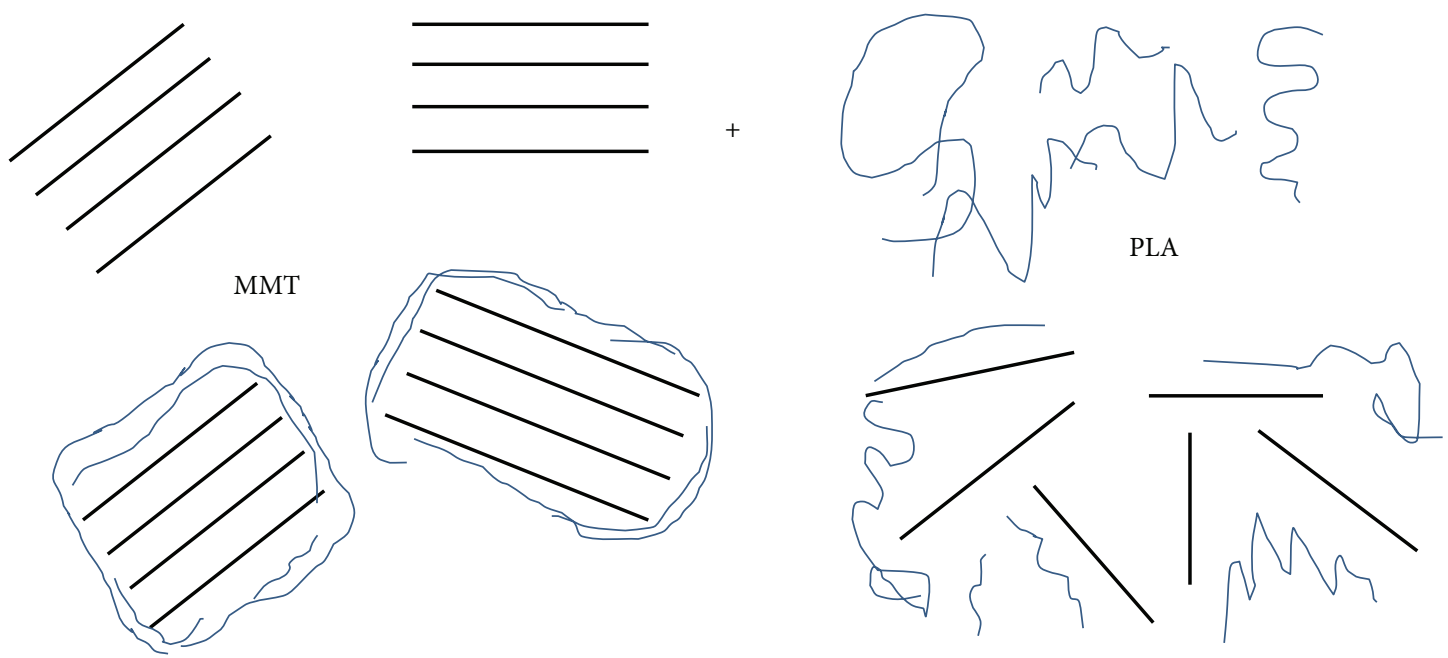

(1) Intercalated PLA/MMT nanocomposites with free orientation

(1) Exfoliated PLA/MMT nanocomposites with free orientation
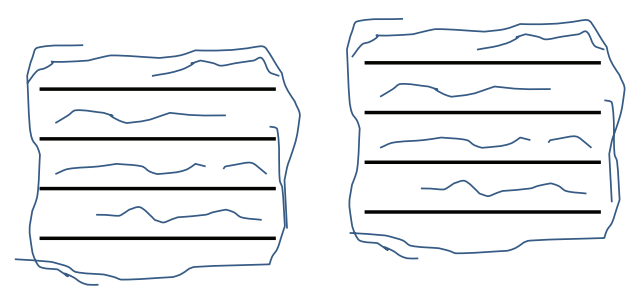

(2) Intercalated PLA/MMT nanocomposites in the direction of flow

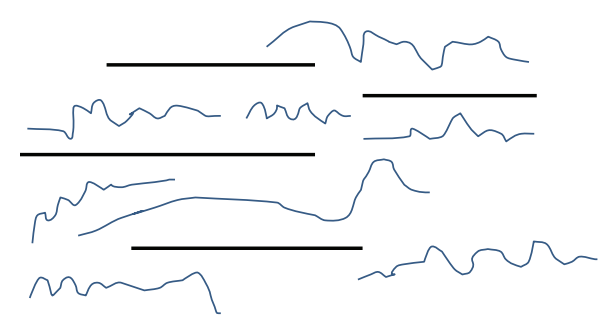

(2) Exfoliated PLA/MMT nanocomposites in the direction of flow

FIGURE 14: Schematic representation of MMT lamellae in PLA nanocomposites with free orientation (1) and in the direction of flow (2).

behaviour is enhanced. The effect in this case may be caused by the presence of a high number of stable covalent bonds between polymer macromolecules and the silane that at low shears create a sort of network responsible for increasing viscosity [48]. At higher shears the different surface energy of unmodified MMT and PLA creates aggregation between MMT nanoparticles and generates small voids that slightly contribute to building up viscosity for unmodified samples; when silane is present, bonds between polymer and silane act as compatibilizer and prevent the aggregation of MMT and the formation of interruptions, lowering melt viscosity.

This variation in melt viscosity is obtained thanks to the improved compatibility between MMT and the polymeric matrix and thanks to the quantity of MMT platelets. Besides, the increase is correlated with the structure of nanocomposites. In fact, high melt viscosities are obtained in polymer nanocomposites with fully exfoliated clay minerals with high aspect ratio that orient lamellae in the direction of flow [49], as reported in Figure 14.

\section{Conclusions}

PLA nanocomposites were synthesized via "in situ” bulk ROP polymerization of LL-lactide with MMT. An epoxy silane was used to modify the surface of MMT and to increase the compatibility between PLA matrix and nanoparticles. Thanks to surface modification and in situ synthesis, very low amounts of nanoparticles (even $0.1 \% \mathrm{w} / \mathrm{w}$ MMT) are enough to get improvements previously observed with far higher quantities of clay ( $5 \% \mathrm{w} / \mathrm{w}$ or more), especially regarding melt viscosity. When MMT with no silane is used, $\overline{M n}$ of the polymers drops even with low quantity of mineral, but when silane is present molecular weights become even higher than those of standard PLA and dispersion of the nanoparticles in the polymeric matrix is favoured. Nanoparticles slightly enhance crystallinity of the polymers $\left(T_{c}\right.$ and $\left.T_{c c}\right)$, especially promoting melt crystallization in comparison to standard PLA and very small quantity of MMT is required when silane is present $(0.1 \% \mathrm{w} / \mathrm{w})$. TGA analyses show that when MMT modified with $15 \% \mathrm{w} / \mathrm{w}$ of organosilane is present, nanocomposites have a thermal stability $\left(T_{1 \%}\right)$ that becomes almost $50^{\circ} \mathrm{C}$ higher than that of standard sample.

The rheological behaviours of nanocomposites show dependence both on silane and MMT content. It is important to underline that, thanks to in situ synthesis, even $0.1 \% \mathrm{w} / \mathrm{w}$ of MMT allows obtaining zero shear viscosity values three times higher than those of standard PLA $\left(\eta_{0}=3.31 * 10^{3}\right.$ for PLA3, versus $1.19 * 10^{3}$ of STD sample) and that when silane is present, $\eta_{0}$ can be even 50 times higher than that of standard PLA thanks to the reaction between the silane present on 
the surface of nanoparticles and polymeric chains, creating a network that increases viscosity; at higher shear rates, high quantities of silane contribute to giving a marked shear thinning behaviour, because it avoids the aggregation of nanoparticles and the formation of voids between the clay and the polymer.

The overall results clearly indicate that combining the use of "in situ" synthesis that allows long contact times between mineral nanoparticles and the growing polymeric chains of PLA together with a coupling agent on the surface of MMT that can react with the polymer can result in very positive effects on many different properties of PLA nanocomposites, even with very low quantities of mineral nanoparticle, that could open new application fields for such nanocomposites. Further studies will be conducted, especially on the samples that showed the higher melt viscosity and thermal stability, to assess their potential for the production of films for packaging and disposables.

\section{Conflict of Interests}

The authors declare that there is no conflict of interests regarding the publication of this paper.

\section{Acknowledgments}

This work has been carried out thanks to the financial support of Fondazione Cariplo and Regione Lombardia for the project "BIOPLANT" given according to the three guidelines: Action 1: "grant for actions regarding industrial research and experimental development finalized to improve competence centers having a regional feature," Action 2: "call for the presentation of projects dedicated to improve the knowledge of scientists in the research field and for supporting the mobility of researchers through highly innovative international projects," and Action 3: "promotion of international projects dedicated to the enhancement of the attractiveness of regional research." The authors wish to thank Professor Francesco Demartin (Dipartimento di Chimica, University of Milan) for the WAXS analyses.

\section{References}

[1] S. Jacobsen, H. G. Fritz, P. Degée, P. Dubois, and R. Jérôme, "Single-step reactive extrusion of PLLA in a corotating twinscrew extruder promoted by 2-ethylhexanoic acid tin(II) salt and triphenylphosphine," Polymer, vol. 41, no. 9, pp. 3395-3403, 2000.

[2] M. Vert, G. Schwarch, and J. Coudane, "Present and future of PLA polymers," Journal of Macromolecular Science, Part A: Pure and Applied Chemistry, vol. 32, pp. 787-796, 1995.

[3] J. A. Cicero, J. R. Dorgan, J. Janzen, J. Garrett, J. Runt, and J. S. Lin, "Supramolecular morphology of two-step, melt-spun poly(lactic acid) fibers," Journal of Applied Polymer Science, vol. 86, no. 11, pp. 2828-2838, 2002.

[4] N. Ogata, G. Jimenez, H. Kawai, and T. Ogihara, "Structure and thermal/mechanical properties of poly(l-lactide)-clay blend," Journal of Polymer Science Part B: Polymer Physics, vol. 35, no. 2, pp. 389-396, 1997.
[5] J. S. Ma, Z. N. Qi, and Y. L. Hu, "Synthesis and characterization of polypropylene/clay nanocomposites," Journal of Applied Polymer Science, vol. 82, no. 14, pp. 3611-3617, 2001.

[6] X. Fu and S. Qutubuddin, "Polymer-clay nanocomposites: exfoliation of organophilic montmorillonite nanolayers in polystyrene," Polymer, vol. 42, no. 2, pp. 807-813, 2001.

[7] J. R. Dorgan, H. Lehermeier, and M. Mang, "Thermal and rheological properties of commercial-grade poly(lactic acids)s," Journal of Polymers and the Environment, vol. 8, no. 1, pp. 1-9, 2000.

[8] P. Meneghetti, S. Shaikh, S. Qutubuddin, and S. Nazarenko, "Synthesis and characterization of styrene-butadiene rubberclay nanocomposites with enhanced mechanical and gas barrier properties," Rubber Chemistry and Technology, vol. 81, no. 5, pp. 821-841, 2008.

[9] R. Singh, J. Pandey, D. Rutot, P. Degée, and P. Dubois, "Biodegradation of $\operatorname{poly}(\varepsilon$-caprolactone $) /$ starch blends and composites in composting and culture environments: the effect of compatibilization on the inherent biodegradability of the host polymer," Carbohydrate Research, vol. 338, no. 17, pp. 1759-1769, 2003.

[10] M. Biswas and S. Sinha Ray, "Recent progress in synthesis and evaluation of polymer montmorillonite nanocomposites," Advances in Polymer Technology, vol. 14, pp. 155-167, 2001.

[11] M. Pluta, J. K. Jeszka, and G. Boiteux, "Polylactide/montmorillonite nanocomposites: structure, dielectric, viscoelastic and thermal properties," European Polymer Journal, vol. 43, no. 7, pp. 2819-2835, 2007.

[12] J.-H. Chang, Y. U. An, and G. S. Sur, "Poly(lactic acid) nanocomposites with various organoclays. I. Thermomechanical properties, morphology, and gas permeability," Journal of Polymer Science, Part B: Polymer Physics, vol. 41, no. 1, pp. 94-103, 2002.

[13] K. Hiltunen, J. V. Seppala, and M. Harkonen, "Lactic acid based poly (ester-urethanes): use of hydroxyl terminated prepolymer in urethane synthesis," Journal of Applied Polymer Science, vol. 63, no. 8, pp. 1091-1100, 1997.

[14] R. A. Vaia and E. P. Giannelis, "Lattice model of polymer melt intercalation in organically-modified layered silicates," Macromolecules, vol. 30, no. 25, pp. 7990-7999, 1997.

[15] A. Zhu, H. Diao, Q. Rong, and A. Cai, "Preparation and properties of polylactide-silica nanocomposites," Journal of Applied Polymer Science, vol. 116, no. 5, pp. 2866-2873, 2010.

[16] Y. Li, R. Cui, L. Ding et al., "How catalysts affect the growth of single-walled carbon nanotubes on substrates," Advanced Materials, vol. 22, no. 13, pp. 1508-1515, 2010.

[17] H. M. Lewkowitz-Shpuntoff, M. C. Wen, A. Singh et al., "The effect of organo clay and adsorbed $\mathrm{FeO}_{3}$ nanoparticles on cells cultured on Ethylene Vinyl Acetate substrates and fibers," Biomaterials, vol. 30, no. 1, pp. 8-18, 2009.

[18] S. S. Ray and M. Okamoto, "Polymer/layered silicate nanocomposites: a review from preparation to processing," Progress in Polymer Science, vol. 28, no. 11, pp. 1539-1641, 2003.

[19] M. Jollands and R. K. Gupta, "Effect of mixing conditions on mechanical properties of polylactide/montmorillonite clay nanocomposites," Journal of Applied Polymer Science, vol. 118, no. 3, pp. 1489-1493, 2010.

[20] M. Mihai, M. A. Huneault, B. D. Favis, and H. Li, "Extrusion foaming of semi-crystalline PLA and PLA/thermoplastic starch blends," Macromolecular Bioscience, vol. 7, no. 7, pp. 907-920, 2007.

[21] Y. Yildirim and A. Oral, "The influence of $\gamma$-ray irradiation on the thermal stability and molecular weight of poly(L-lactic acid) 
and its nanocomposites," Radiation Physics and Chemistry, vol. 96, pp. 69-74, 2014.

[22] S. M. Lai, S. H. Wu, G. G. Lin, and T. M. Don, "Unusual mechanical properties of melt-blended poly(lactic acid) (PLA)/clay nanocomposites," European Polymer Journal, vol. 52, pp. 193206, 2014.

[23] S. I. Pirani, P. Krishnamachari, and R. Hashaikeh, "Optimum loading level of nanoclay in PLA nanocomposites. Impact on the mechanical properties and glass transition temperature," Journal of Thermoplastic Composite Materials, vol. 27, no. 11, pp. 1461-1478, 2014.

[24] B. Ayana, S. Suin, and B. B. Khatua, "Highly exfoliated ecofriendly thermoplastic starch (TPS)/poly (lactic acid)(PLA)/ clay nanocomposites using unmodified nanoclay," Carbohydrate Polymers, vol. 110, pp. 430-439, 2014.

[25] N. Ogata, G. Jimenez, H. Kawai, and T. Ogihara, "Structure and thermal/mechanical properties of poly(l-lactide)-clay blend," Journal of Polymer Science, Part B: Polymer Physics, vol. 35, no. 2, pp. 389-396, 1997.

[26] N. Tsubokawa, K. Maruyama, Y. Sone, and M. Shimomura, "Graft polymerization of acrylamide from ultrafine silica particles by use of a redox system consisting of ceric ion and reducing groups on the surface," Polymer Journal, vol. 21, no. 6, pp. 475481, 1989.

[27] L. Basilissi, G. D. Silvestro, H. Farina, and M. A. Ortenzi, "Synthesis and characterization of PLA nanocomposites containing nanosilica modified with different organosilanes I. Effect of the organosilanes on the properties of nanocomposites: macromolecular, morphological, and rheologic characterization," Journal of Applied Polymer Science, vol. 128, no. 3, pp. 1575-1582, 2013.

[28] F. Ublekov, J. Baldrian, J. Kratochvil, M. Steinhart, and E. Nedkov, "Influence of clay content on the melting behavior and crystal structure of nonisothermal crystallized poly(L-lactic acid)/nanocomposites," Journal of Applied Polymer Science, vol. 124, no. 2, pp. 1643-1648, 2012.

[29] J. F. Turner II, A. Riga, A. O’Connor, J. Zhang, and J. Collis, "Characterization of drawn and undrawn poly-L-lactide films by differential scanning calorimetry," Journal of Thermal Analysis and Calorimetry, vol. 75, no. 1, pp. 257-268, 2004.

[30] L. Cabedo, D. Plackett, E. Giménez, and J. M. Lagarón, “Studying the degradation of polyhydroxybutyrate-co-valerate during processing with Clay-Based nanofillers," Journal of Applied Polymer Science, vol. 112, no. 6, pp. 3669-3676, 2009.

[31] K. Woong Kim and S. I. Woo, "Synthesis of high-molecularweight poly(L-lactic acid) by direct polycondensation," Macromolecular Chemistry and Physics, vol. 203, no. 15, pp. 2245-2250, 2002.

[32] A. Du, G. A. Gelves, D. Koo, U. Sundararaj, and R. Cairncross, "Water transport in polylactide and polylactide/montmorillonite composites," Journal of Polymers and the Environment, vol. 21, no. 1, pp. 8-15, 2013.

[33] H. J. Ploehn and C. Liu, "Quantitative analysis of montmorillonite platelet size by atomic force microscopy," Industrial and Engineering Chemistry Research, vol. 45, no. 21, pp. 7025-7034, 2006.

[34] X. Wen, Y. Lin, C. Han, L. Han, Y. Li, and L. Dong, "Dramatic improvements in mechanical properties of poly(L-lactide)/ silica nanocomposites by addition of hyperbranched poly(ester amide)," Macromolecular Materials and Engineering, vol. 295, no. 5, pp. 415-419, 2010.
[35] M. Yasuniwa, S. Tsubakihara, K. Iura, Y. Ono, Y. Dan, and K. Takabashi, "A study of the crystallization, melting and foaming behaviors of Polylactic acid in compressed $\mathrm{CO}_{2}$," Polymer, vol. 47, pp. 7554-7563, 2006.

[36] H. Zhou, T. B. Green, and Y. L. Joo, "The thermal effects on electrospinning of polylactic acid melts," Polymer, vol. 47, no. 21, pp. 7497-7505, 2006.

[37] J. Q. Pham, C. A. Mitchell, J. L. Bahr, J. M. Tour, K. Krishanamoorti, and P. F. Green, "Glass transition of polymer/ single-walled carbon nanotube composite films," Journal of Polymer Science Part B: Polymer Physics, vol. 41, no. 24, pp. 33393345, 2003.

[38] B. J. Ash, L. S. Schadler, and R. W. Siegel, "Glass transition behavior of alumina/polymethylmethacrylate nanocomposites," Materials Letters, vol. 55, no. 1-2, pp. 83-87, 2002.

[39] K. Fukushima, D. Tabuani, and G. Camino, "Nanocomposites of PLA and PCL based on montmorillonite and sepiolite," Materials Science and Engineering C, vol. 29, no. 4, pp. 1433-1441, 2009.

[40] L. Basilissi, G. di Silvestro, H. Farina, and M. A. Ortenzi, "Synthesis and characterization of PLA nanocomposites containing nanosilica modified with different organosilanes II: effect of the organosilanes on the properties of nanocomposites: thermal characterization," Journal of Applied Polymer Science, vol. 128, no. 5, pp. 3057-3063, 2013.

[41] I. C. McNeill and H. A. Leiper, "Degradation studies of some polyesters and polycarbonates-2. Polylactide: degradation under isothermal conditions, thermal degradation mechanism and photolysis of the polymer," Polymer Degradation and Stability, vol. 11, no. 4, pp. 309-326, 1985.

[42] F.-D. Kopinke, M. Remmler, K. Mackenzie, M. Möder, and O. Wachsen, "Thermal decomposition of biodegradable polyesters-II. Poly(lactic acid)," Polymer Degradation and Stability, vol. 53, no. 3, pp. 329-342, 1996.

[43] M.-T. Liu, M.-F. Pu, H.-W. Ma, Y.-F. Hu, X.-J. Liu, and X. Pang, "The effect of organic modifier-12-aminolauric acid on morphology and thermal properties of polylactide nanocomposites," Polymer Composites, vol. 32, no. 6, pp. 1002-1008, 2011.

[44] S. Sareeladdanon and P. Potiyaraj, "Mechanical properties of PLA/LLDPE films reinforced with silica from rice husk," Advanced Materials Research, vol. 1025-1026, pp. 221-226, 2014.

[45] X. Wen, K. Zhang, Y. Wang et al., "Study of the thermal stabilization mechanism of biodegradable poly(l-lactide)/silica nanocomposites," Polymer International, vol. 60, no. 2, pp. 202 210, 2011.

[46] S.-Y. Gu, J. Ren, and B. Dong, "Melt rheology of polylactide/montmorillonite nanocomposites," Journal of Polymer Science Part B: Polymer Physics, vol. 45, no. 23, pp. 3189-3196, 2007.

[47] B. Wang, T. Wan, and W. Zeng, "Dynamic rheology and morphology of polylactide/organic montmorillonite nanocomposites," Journal of Applied Polymer Science, vol. 121, no. 2, pp. 1032-1039, 2011.

[48] V. Katiyar and H. Nanavati, "In situ synthesis of high molecular weight poly(l-lactic acid) clay nanocomposites," Polymer Engineering and Science, vol. 51, pp. 2066-2077, 2011.

[49] J. Lagaron and G. Sanchez, Thermoplastic Nanocomposites for Rigid and Flexible Food Packaging Applications, E. Environmentally Friendly Food Packaging, Woodhead Publishers, Boca Raton, Fla, USA, 2008. 

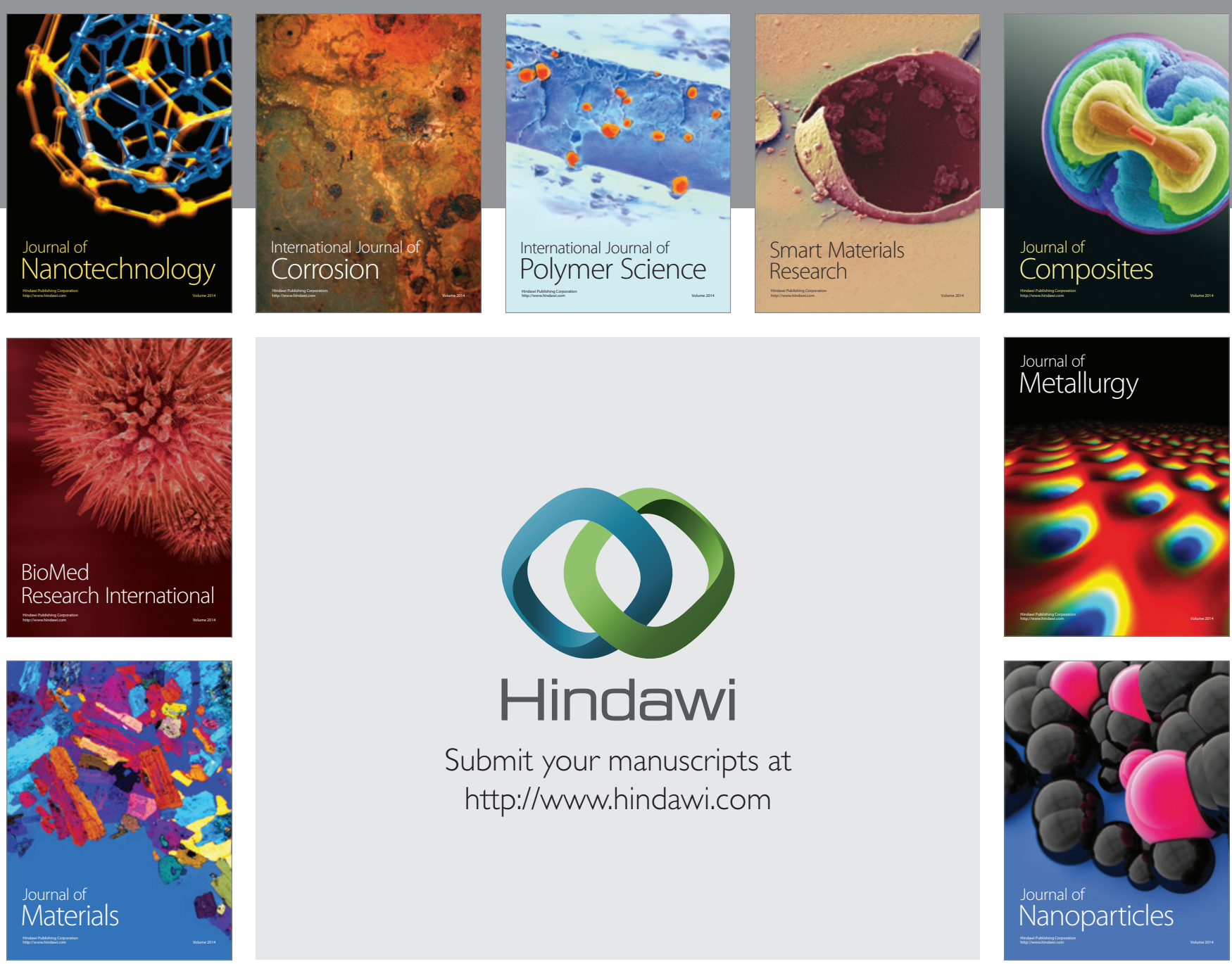

Submit your manuscripts at http://www.hindawi.com
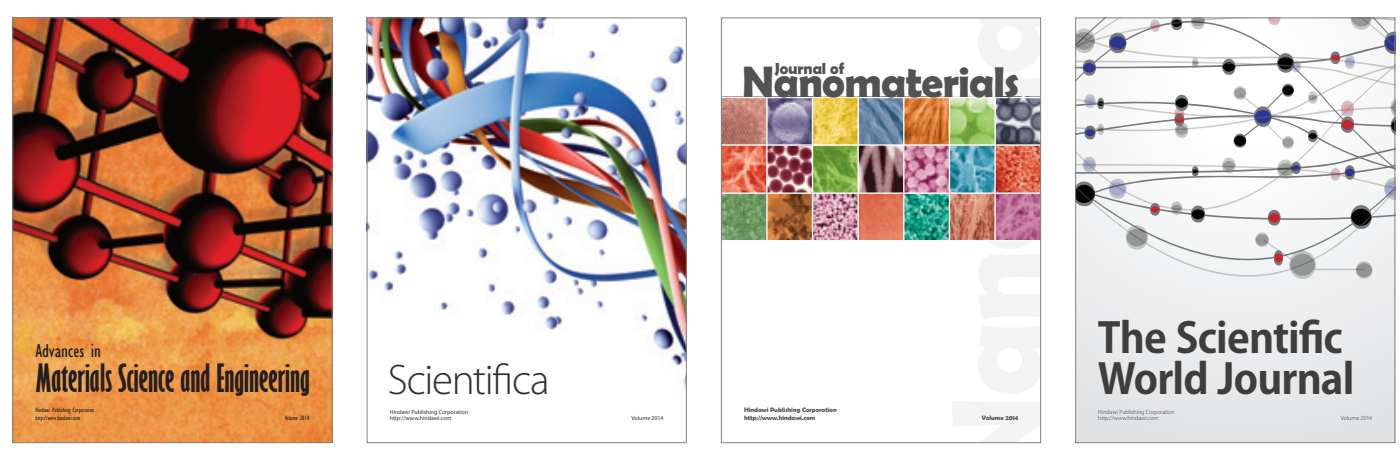

\section{The Scientific World Journal}
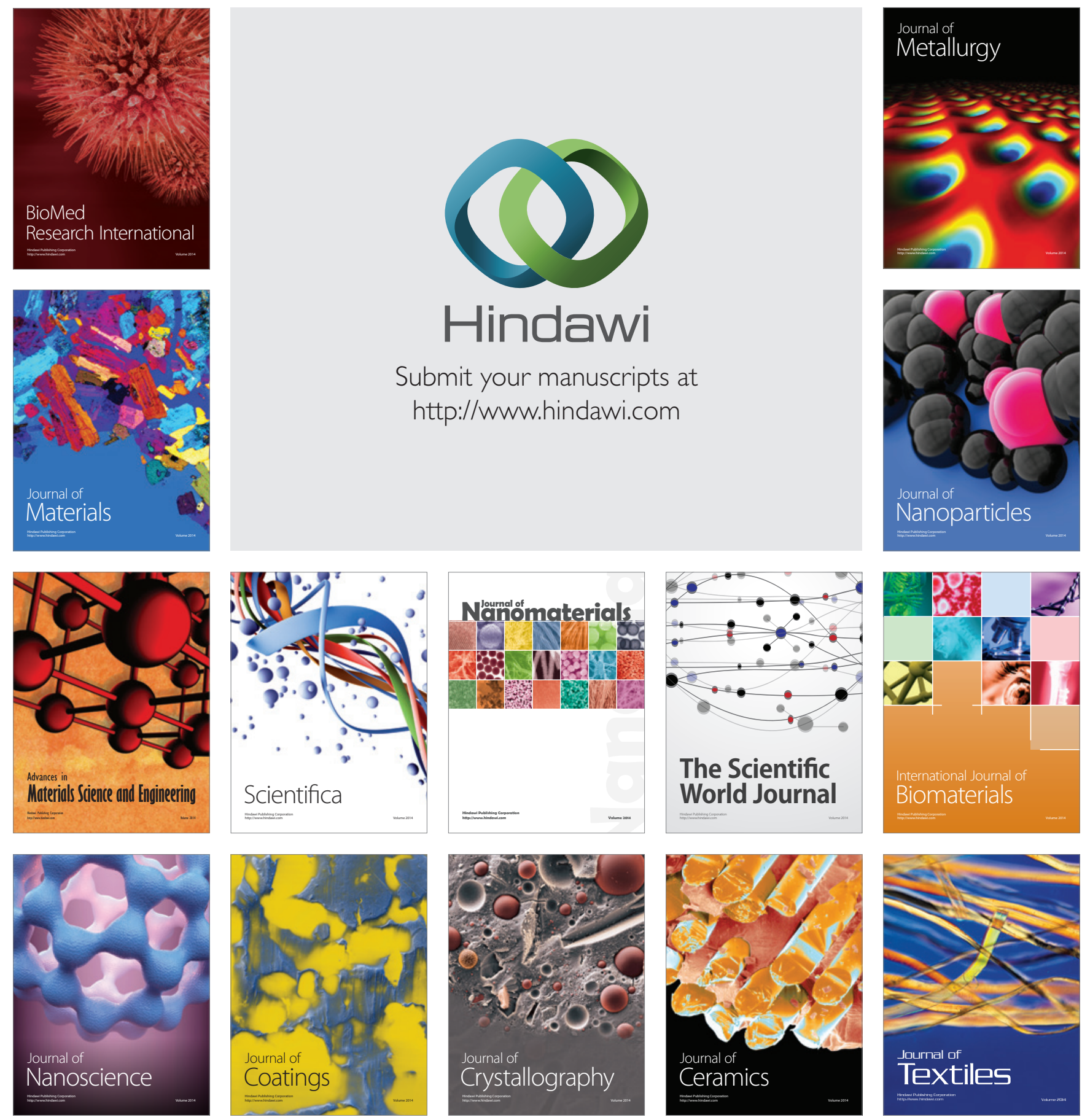